\title{
MURAME parameter setting for creditworthiness evaluation: data-driven optimization
}

\author{
Marco Corazza ${ }^{1}$. Giovanni Fasano ${ }^{2}$. Stefania Funari ${ }^{2} \cdot$ Riccardo Gusso $^{1}$
}

Received: 2 December 2019 / Accepted: 9 February 2021 / Published online: 3 May 2021

(c) The Author(s) 2021

\begin{abstract}
In this paper, we amend a multi-criteria methodology known as MURAME, to evaluate the creditworthiness of a large sample of Italian Small and Medium-sized Enterprises, using as input their balance sheet data. This methodology produces results in terms of scoring and of classification into homogeneous rating classes. A distinctive goal of this paper is to consider a preference disaggregation method to endogenously determine some parameters of MURAME, by solving a nonsmooth constrained optimization problem. Because of the complexity of the involved mathematical programming problem, for its solution we use an evolutionary metaheuristic, coupled with a specific efficient initialization. This is combined with an unconstrained reformulation of the problem, which provides a reasonable compromise between the quality of the solution and the computational burden. An extensive numerical experience is reported, comparing an exogenous choice of MURAME parameters with our approach.
\end{abstract}

Keywords Multi-Criteria Decision Analysis (MCDA) · MUlticriteria RAnking MEthod (MURAME) · Small and Medium-sized Enterprises (SMEs) ·

Creditworthiness evaluation · Preference disaggregation · Particle Swarm

Optimization (PSO)

JEL Classification C61, C63, G29, G33

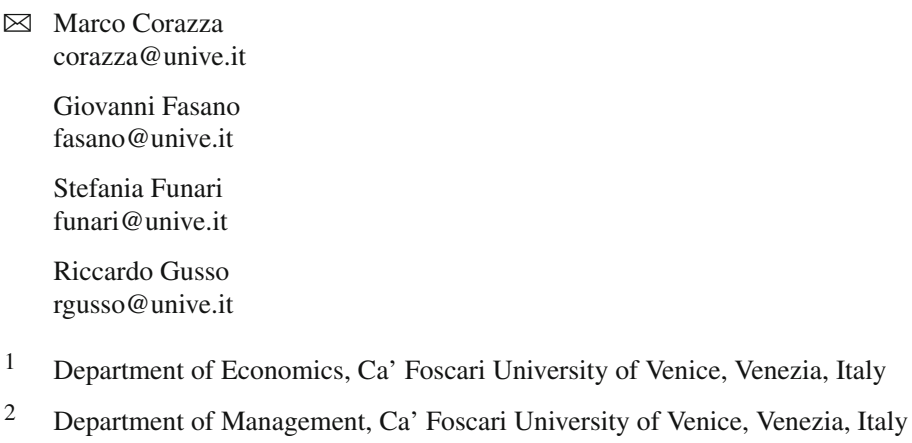




\section{Introduction}

The use of Multi-Criteria Decision Analysis (MCDA) for credit risk modeling has become very common in the specialized literature. MCDA enables to simultaneously consider the many factors that affect firms' creditworthiness, in order to directly involve the decision maker (DM) in the credit risk assessment process, and-particularly important - to incorporate her/his preference structure in the evaluation model. Usually, the preferential system of the DM is mainly specified by the parameters of the adopted MCDA. Of course, the quality of the creditworthiness analysis performed by the considered MCDA strongly depends on the "appropriate" determination of its parameters. When this determination is not immediately available by the DM (as in most cases), it can be endogenously inferred by a proper reference set of evaluations/decisions previously taken by the same DM. The methodological approach considered in this occurrence is the so-called preference disaggregation analysis, which is widely used in different application contexts (see Jacquet-Lagrèze and Siskos 2001 for a classical review and see Kadzinski et al. 2020a, b for recent developments).

Within this reference framework, in this paper we consider a MCDA methodology known as MUlticriteria RAnking MEthod (MURAME) ${ }^{1}$ to evaluate the creditworthiness of a large sample of Italian Small and Medium-sized Enterprises (SME), using as input their balance sheet data. This methodology produces results in terms of scoring and classification into homogeneous rating classes. A distinctive goal of this paper is to consider a preference disaggregation method to endogenously determine some parameters of MURAME, by solving a nonsmooth constrained optimization problem. Because of the complexity of the mathematical programming problem, for its solution we use the evolutionary metaheuristic known as Particle Swarm Optimization (PSO), coupled with a specific efficient initialization. This is combined with an unconstrained reformulation of the problem, which provides a reasonable compromise between the quality of the solution and the computational burden.

The study we present here continues a line of research initially developed in Corazza et al. (2014, 2015b, 2016). Briefly, with reference only to the two papers of main interest for the current study, in Corazza et al. (2015b) a novel evolutionary approach was adopted in order to determine the MURAME parameters. To this aim, an auxiliary mathematical programming problem was solved for tuning MURAME parameters. It was based on minimizing the inconsistency between the creditworthiness evaluations from the MURAME-based methodology and those revealed by a DM. The heuristic procedure PSO was adopted, in order to solve an unconstrained global optimization problem. In particular, PSO was adopted for the minimization of a nondifferentiable penalty function, where standard initializations for PSO were used, inasmuch as the main focus of the paper was on a creditworthiness problem. In Corazza et al. (2016), a general MURAME-based methodology has been applied to evaluate the creditworthiness of about 36,500 Italian SMEs in the period 2006-2008, a triennium including the beginning of the past economic and financial crisis. In that paper, the MURAMEbased procedure was not coupled with any optimization procedure, and the assessment

\footnotetext{
1 MURAME has been originally proposed for the assessment of industrial projects (see Goletsis et al. 2001, 2003).
} 
of its parameters was exogenously based on standard indications from the literature. The obtained results have shown that the MURAME-based methodology was able to detect early signals of recession in the Italian SMEs sector. Furthermore, numerical results have also confirmed the robustness of this methodology with respect to the number of the evaluation criteria.

Despite the findings obtained in Corazza et al. (2015b, 2016), one of the key aspects to improve efficiency of MURAME is represented by a reliable and effective assessment set of its parameters. Even though the approach in Corazza et al. (2015b) partially focuses on an endogenous assessment of MURAME parameters by PSO, it requires to force the reformulation of a constrained optimization problem into an unconstrained one, through the use of penalty terms. This might be a difficult task, since the use of penalty terms can possibly raise numerical instabilities, as better detailed in the sequel.

Conversely, the current contribution has three distinctive tasks:

(i) We employ an endogenous computation of some MURAME parameters to score and to classify into homogeneous rating classes the Italian SMEs belonging to the same dataset of firms considered in Corazza et al. (2016). This is an enhancement to what we have done in Corazza et al. (2016), where the scoring and the classification were determined only using an exogenous specification of the MURAME parameters. Notice that the endogenous determination employed in the present work is focused on correctly scoring and classifying bankrupt firms, which are particularly important for any economic/financial institution. (The reasons of this choice will be detailed in Sect. 4.) In turn, this is different from what some of these authors have done in Corazza et al. (2015b), where the MURAME parameters were endogenously determined to minimize the number of all the firms, both active and bankrupt, incorrectly classified;

(ii) The preference disaggregation method we consider to endogenously determine some parameters of MURAME implies to solve a constrained optimization problem. But the metaheuristic solution we use was conceived for unconstrained problems, and its direct application cannot prevent from generating infeasible solutions. Therefore, in order to manage the constraints of the involved mathematical programming problem, in place of adopting the penalty function approach applied in Corazza et al. (2013, 2015b), we consider a novel nonlinear reformulation of the constrained optimization problem in terms of an unconstrained one ${ }^{2}$;

(iii) We use the metaheuristic procedure as in Corazza et al. (2015b), but we endow it with two recently introduced deterministic initializations (see Corazza et al. 2015a; Diez et al. 2016) never used before in this research area. We remark that the initialization procedures adopted here significantly differ from the one in Campana et al. (2010), as better detailed in Subsect. 5.3. Finally, to validate our approach, we compare the results in terms of scoring and of classification, when comparing the above initialization procedures and the standard random initialization in Corazza et al. (2015b) (see Sect. 6). Furthermore, we also

\footnotetext{
2 Notice that by doing so we can maintain PSO as in its original iteration.
} 
carry out a comparison with the results got by applying our MURAME-based creditworthiness model with the exogenous parameterization considered in Corazza et al. (2016) (see again Sect. 6).

The remainder of this paper is organized as follows. The next section reports a brief review of the literature, whose main purpose is not to be exhaustive but, rather, to highlight the most relevant contributions on this research area. Sect. 3 presents the MURAME methodology adopted for the creditworthiness evaluation of SMEs. Section 4 first formulates the constrained optimization problem for the endogenous determination of the MURAME parameters, then describes the balance sheet data of the Italian SMEs used in the empirical investigation. Regarding Sect. 5: it first introduces some basics about PSO, then it illustrates the nonlinear reformulation of the constrained optimization problem in terms of an unconstrained one. Next, to compare the PSO solver based on this novel reformulation with the PSO one based on the penalty approach in Corazza et al. (2013, 2015a), Sect. 5 briefly presents the unconstrained reformulation of the mathematical programming problem within a penalty framework. Lastly, the same section details the PSO deterministic initializations we use. Section 6: first presents the results related to the classification of the investigated Italian SMEs into homogeneous rating classes, using the methodology we propose here; then compares these results with those obtained in Corazza et al. (2016) using an exogenous determination of the MURAME parameters. Section 7: first compares the aforementioned results also with those from a PSO solver based on a penalty function approach; then issues some very preliminary ideas to possibly improve the exploitation of the informative content coming from the aforementioned SMEs. Finally, Sect. 8 includes some concluding remarks.

\section{Brief review of the literature}

The topic of creditworthiness evaluation of SMEs has been mainly addressed by using statistical and econometric techniques. Some of the recent contributions on this topic have been stimulated by the Basel capital accord Basel II (see Basel Committee on Banking Supervision 2004), that permitted banks to distinguish separately the exposures to SMEs. The effects of Basel II on the bank capital requirements have been investigated in Altman and Sabato (2005) in case of US, Italian and Australian SMEs.

In particular, we highlight the importance of modeling credit risk peculiarly for SMEs. This issue has received an increasing attention: papers (Altman and Sabato 2005, 2007; Altman et al. 2010), and the very recent contribution (Altman et al. 2020) concerning the Italian SMEs, have explicitly pointed out the importance to develop credit risk models specifically for SMEs. Particularly, Altman and Sabato (2007) identified a set of financial ratios that could influence SMEs creditworthiness. It showed the superiority of the proposed model in terms of default prediction accuracy, compared to a generic corporate model.

It is interesting to note that over the years new methodologies have attracted the attention of creditworthiness assessment. For instance, the early paper (Altman et al. 1994) compares traditional statistical methodologies for distress classification and 
prediction, such as linear discriminant analysis, with neural networks, and applied the results to retailing/trading and construction firms in Italy. Moreover, Akkoç (2012) proposed a credit scoring model that simultaneously uses an artificial neural network and fuzzy logic, whereas several contributions explored the use of evolutionary computing for credit scoring (see Marqués et al. 2013 for an extensive literature review on the evolutionary computation methods applied to credit scoring models).

It is also possible to observe a growing interest toward the MCDA-based tools capable of providing effective support to creditworthiness evaluation (see for instance Baourakis et al. 2009; Doumpos and Figueira 2019; Doumpos et al. 2002; Doumpos and Pasiouras 2005; Doumpos and Zopounidis 2011; García et al. 2013; Khalil et al. 2000; Mousavi and Ouenniche 2018). Among them, a few studies have specifically addressed the issue of credit risk evaluation of SMEs through MCDA. Two main research directions can be observed on this last topic: the one using the MURAME methodology to evaluate SMEs creditworthiness (see Corazza et al. 2014, 2015b, 2016) and the one proposing ELECTRE TRI-based methods for the creditworthiness evaluation of innovative SMEs (see Angilella and Mazzú 2015, 2019).

We remark that the present contribution is part of the first line of research, as it is inspired by Corazza et al. (2015b, 2016), that detail the use of MURAME to deal with a creditworthiness evaluation problem (the differences with the two previous studies have been highlighted in the introductory section).

We conclude this section by noting that, despite the broad literature on MCDA applications, there are relatively few applications of MCDA to the credit risk assessment of SMEs. However, most of the studies mentioned above on this topic have been proposed in recent years, so that research in this field appears promising.

\section{MURAME for creditworthiness evaluation}

In order to assess SME creditworthiness, we use an approach that jointly obtains a ranking of SMEs on the basis of multiple indicators, typically balance sheet indicators, and classifies the enterprises into homogeneous classes with respect to their creditworthiness.

Let us denote by $A=\left\{a_{1}, \ldots, a_{m}\right\}$ the set of firms whose creditworthiness has to be evaluated on the basis of the set of indicators $\left\{I_{1}, \ldots, I_{n}\right\}$ and by $G=\left[g_{i j}\right]$ the decision matrix of dimension $m \times n$ in which the entry $g_{i j}$ indicates the performance of firm $a_{i}$ with respect to indicator $I_{j}$. A weight $w_{j}$, with $j=1, \ldots, n$, is also associated to each $I_{j}$, being $\|\mathbf{w}\|_{1}=1$, indicating the relative importance of each indicator in the evaluation process.

The approach based on MURAME adopted for creditworthiness evaluation is implemented in three steps (see Corazza et al. 2016). The objective of Step I is to build the so-called reference profiles to use for classifying firms into homogeneous rating classes. Regarding Steps II and III, since MURAME results from merging two common multicriteria methods, ELECTRE III (see Roy 1968) and PROMETHEE II (see Brans and Vincke 1985), they are peculiar of its two "ancestors." In particular, Step II aims at computing concordance and discordance indicators, which in turn are used to build the outranking index $O\left(a_{i}, a_{k}\right)$ for each pair of firms $\left(a_{i}, a_{k}\right)$. The outranking 
relation between $a_{i}$ and $a_{k}$ allows to judge whether there are enough arguments to definitely state that firm $a_{i}$ is at least as good as firm $a_{k}$ (see for example (Belton and Stewart 2002, ch. 8) for the definition of the outranking relation and the description of the so-called outranking methods in MCDA). Lastly, Step III uses the outranking indexes in order to compute a final score associated to each firm, which enables us to completely rank the firms.

\subsection{Step I: reference profiles}

The first step aims at specifying the reference profiles to add to the firms, for classifying them into homogeneous classes from the point of view of their creditworthiness. Reference profiles represent a sort of benchmark profiles related to fictitious firms, which could also be specified by the DM. They delimit contiguous rating classes and serve as comparison with the profiles of real firms. Notice that, if we need to classify firms in $l$ rating classes Class $_{1}, \ldots$, Class $_{l}$, a number of $l-1$ reference profiles has to be considered.

Let us denote by $\mathcal{R}=\left\{r_{1}, \ldots, r_{l-1}\right\}$ the set of reference profiles and by $\tilde{A}=A \cup \mathcal{R}$ the union of the set of firms and $\mathcal{R}$ itself; the elements of $\tilde{A}$ are denoted by $\tilde{a}_{i}$, with $i=1, \ldots, m+l-1$.

The final classification of firm $a_{i} \in A$ is determined according with this procedure:

$$
a_{i} \in \text { Class }_{j} \quad \Longleftrightarrow \varphi\left(r_{j-1}\right)>\varphi\left(a_{i}\right) \geq \varphi\left(r_{j}\right)
$$

for $j=1, \ldots, l$, where $\varphi(\cdot)$ is the final score associated by MURAME to the considered alternative ${ }^{3}$, with $\varphi\left(r_{0}\right)=+\infty$ and $\varphi\left(r_{l}\right)=-\infty$.

To compute the reference profiles, we follow the method adopted in Corazza et al. (2016), with the only difference that we assume five rating classes instead of ten. This choice agrees with a recent proposal of the Italian Ministry of Economic Development, regarding a guarantee fund for SMEs, which indeed considers an evaluation model based on five creditworthiness classes. In particular, for each indicator $I_{j}$, we first calculate the sample quintiles of its empirical distribution, that we named $I_{j}^{1}, I_{j}^{2}, \ldots, I_{j}^{4}$; then, we aggregate the quintiles of the same order, obtaining 4 fictitious alternatives to be used as reference profiles:

$$
\left\{\begin{array}{c}
r_{1}=\left(I_{1}^{1}, I_{2}^{1}, \ldots, I_{n}^{1}\right) \\
\vdots \\
r_{4}=\left(I_{1}^{4}, I_{2}^{4}, \ldots, I_{n}^{4}\right) .
\end{array}\right.
$$

It is noteworthy that such reference profiles produce homogeneous rating classes whose cardinalities strictly depend on the features of the empirical distributions of the indicators.

\footnotetext{
3 A formal definition of $\varphi(\cdot)$ is provided in (6).
} 


\subsection{Step II: concordance, discordance and outranking indexes}

The construction of concordance and discordance indexes in MURAME is based on a quite realistic preference structure. Indeed, with respect to the creditworthiness, this approach permits the DM to express a strong preference for a certain firm, or to consider two firms indifferent, or even to exhibit his/her indecision through a weak preference for a given firm.

The use of preference and indifference thresholds related to each indicator $I_{j}$, which are denoted by $p_{j}$ and $q_{j}$, respectively, with $0 \leq q_{j} \leq p_{j}$, allows to differentiate between the three preference zones described above.

Let us consider indicator $I_{j}$ and compare the pair of firms $\left(\tilde{a}_{i}, \tilde{a}_{k}\right)$ with respect to such an indicator. In particular, if $g_{k j} \geq g_{i j}+p_{j}$, then there is a strict preference for the second firm $\tilde{a}_{k}$. More generally, the following local concordance index $C_{j}\left(\tilde{a}_{i}, \tilde{a}_{k}\right)$ can be introduced to summarize the elements in favor of the first firm $\tilde{a}_{i}$ over firm $\tilde{a}_{k}$ :

$$
C_{j}\left(\tilde{a}_{i}, \tilde{a}_{k}\right)=\left\{\begin{array}{cl}
1 & \text { if } g_{k j} \leq g_{i j}+q_{j} \\
0 & \text { if } g_{k j} \geq g_{i j}+p_{j} \\
\frac{g_{i j}-g_{k j}+p_{j}}{p_{j}-q_{j}} & \text { otherwise. }
\end{array}\right.
$$

Notice that the local concordance index reaches its maximum value, i.e., $C_{j}\left(\tilde{a}_{i}, \tilde{a}_{k}\right)=1$, when the second firm $\tilde{a}_{k}$ is not preferred to the first one, whereas in the intermediate zone of indecision the index takes values in the interval $(0,1)$, and it (linearly) decreases as $g_{k j}-g_{i j}$ increases.

On the other hand, a discordance principle represents the reasons against the statement that firm $\tilde{a}_{i}$ is at least as good as firm $\tilde{a}_{k}$. The following local discordance index can be introduced:

$$
D_{j}\left(\tilde{a}_{i}, \tilde{a}_{k}\right)=\left\{\begin{array}{cl}
0 & \text { if } g_{k j} \leq g_{i j}+p_{j} \\
1 & \text { if } g_{k j} \geq g_{i j}+v_{j} \\
\frac{g_{k j}-g_{i j}-p_{j}}{v_{j}-p_{j}} & \text { otherwise, }
\end{array}\right.
$$

where the parameter $v_{j}$, with $v_{j} \geq p_{j}$, indicates the veto threshold. When $g_{k j} \geq$ $g_{i j}+v_{j}$, one has that the second firm $\tilde{a}_{k}$ performs strongly better than firm $\tilde{a}_{i}$ with respect to indicator $I_{j}$, so that the local discordance index $D_{j}\left(\tilde{a}_{i}, \tilde{a}_{k}\right)$ reaches its maximal value of 1 . Otherwise, when $g_{k j} \leq g_{i j}+p_{j}$, one has that the firm $\tilde{a}_{k}$ performs approximately as the firm $\tilde{a}_{i}$, so that the index reaches its minimal value of 0 , and the indicator $I_{j}$ does not put any veto. In the weak preference zone, $D_{j}\left(\tilde{a}_{i}, \tilde{a}_{k}\right)$ shows a (linear) increase as $g_{k j}-g_{i j}$ increases.

Lastly, in order to build the outranking index $O\left(\tilde{a}_{i}, \tilde{a}_{k}\right)$ for the pair $\left(\tilde{a}_{i}, \tilde{a}_{k}\right)$, both concordance and discordance indexes have to be simultaneously considered, as in:

$$
O\left(\tilde{a}_{i}, \tilde{a}_{k}\right)=\left\{\begin{array}{cl}
C\left(\tilde{a}_{i}, \tilde{a}_{k}\right) & \text { if } D_{j}\left(\tilde{a}_{i}, \tilde{a}_{k}\right) \leq C\left(\tilde{a}_{i}, \tilde{a}_{k}\right) \quad \forall j \\
C\left(\tilde{a}_{i}, \tilde{a}_{k}\right) \prod_{j \in T} \frac{1-D_{j}\left(\tilde{a}_{i}, \tilde{a}_{k}\right)}{1-C\left(\tilde{a}_{i}, \tilde{a}_{k}\right)} \text { otherwise, }
\end{array}\right.
$$


where $C\left(\tilde{a}_{i}, \tilde{a}_{k}\right)=\sum_{j=1}^{n} w_{j} C_{j}\left(\tilde{a}_{i}, \tilde{a}_{k}\right)$ is the global concordance index, which aggregates all the local ones using the relative weights associated to the indicators, and $T \subseteq\{1, \ldots, n\}$ denotes the subset of indicators which satisfy the condition $D_{j}\left(\tilde{a}_{i}, \tilde{a}_{k}\right)>C\left(\tilde{a}_{i}, \tilde{a}_{k}\right)$. Broadly speaking, $O\left(\tilde{a}_{i}, \tilde{a}_{k}\right)$ is higher when there are enough arguments to state that firm $\tilde{a}_{i}$ is at least as good as firm $\tilde{a}_{k}$, i.e. when $C_{j}\left(\tilde{a}_{i}, \tilde{a}_{k}\right)$ is high, while there are no particular reasons to reject this assertion, i.e. when $D_{j}\left(\tilde{a}_{i}, \tilde{a}_{k}\right)$ are low. On the contrary, if the performance of a firm $\tilde{a}_{i}$ with respect to at least an indicator $I_{j}$ is rather low, i.e. if at least some $D_{j}\left(\tilde{a}_{i}, \tilde{a}_{k}\right)$ is high, the outranking index decreases. Observe that $O\left(\tilde{a}_{i}, \tilde{a}_{k}\right)$ can even vanish in case of maximum discordance, i.e. $D_{j}\left(\tilde{a}_{i}, \tilde{a}_{k}\right)=1$, for even only one indicator.

\subsection{Step III: net flow and firms ranking}

By using the outranking indexes $O\left(\tilde{a}_{i}, \tilde{a}_{k}\right)$ obtained in the previous step, the final score, or net flow, $\varphi\left(\tilde{a}_{i}\right)$ is computed for each firm $\tilde{a}_{i}$, as follows:

$$
\varphi\left(\tilde{a}_{i}\right)=\sum_{i \neq k} O\left(\tilde{a}_{i}, \tilde{a}_{k}\right)-\sum_{i \neq k} O\left(\tilde{a}_{k}, \tilde{a}_{i}\right) .
$$

Broadly speaking, the first summation gives the aggregation of how much firm $\tilde{a}_{i}$ outranks with respect to any other firm, whereas the second summation provides the aggregation of how much any other firm outranks with respect to firm $\tilde{a}_{i}$.

Lastly, the final score (6) is employed to rank firms from the best to the worst.

In conclusion, we highlight that the outputs produced by our MURAME-based approach for creditworthiness evaluations are the (relative) scoring and ranking of the investigated firms, and their classification into homogeneous rating classes. On the basis of these outputs, other important quantities for credit risk assessment can be calculated, like for instance the probabilities of default and of migration (see Corazza et al. 2014), and various model's performance metrics. However, the calculation of such quantities is beyond the scope of this paper. As a matter of fact, the main target of our research is to improve as much as possible the produced outputs by addressing an appropriate preference disaggregation problem (see the next section). Of course, this improvement also leads to an enhancement of calculation of the quantities above. In particular, notice that by ranking and by classifying firms according to their score, we are implicitly satisfying requirements of common used model's performance metrics in credit risk assessment, as for example the area under the Receiver Operating Characteristic (ROC) curve (see Fawcett 2006).

\section{The optimization problem for the endogenous parameter specification}

As stated in Sect. 1, the initial task of this study is to endogenously determine some MURAME parameters in order to minimize the inconsistency of the firms classification produced by our approach for creditworthiness evaluation. As it will become clear later 
on, the features of the PSO-based optimization algorithm we adopt actually allow only the assessment of some MURAME parameters, and not all of them. Indeed, since PSO is a metaheuristic procedure for global optimization, the computational cost associated with its application strongly increases with the number of decision variables.

At first, our research focuses on the endogenous calculation of the weights $\left\{w_{j}\right\}$. Notice that they likely summarize the main informative parameters on the DM's preference structure as they specify the (relative) importance of the indicators $\left\{I_{j}\right\}$. This is one of the reasons for their choice; other rationales will be detailed later in this section. Regarding the determination of the other parameters, i.e. the thresholds $\left\{p_{j}\right\}$, $\left\{q_{j}\right\}$ and $\left\{v_{j}\right\}$, we adopt the exogenous setting suggested in Corazza et al. (2014). In a few details, assuming without loss of generality that the indicators $I_{j}$ are real numbers in the range $s_{j}=\max I_{j}-\min I_{j}$, for each indicator, the preference, indifference and veto thresholds are, respectively, set as follows:

$$
p_{j}=\frac{2}{3} s_{j}, q_{j}=\frac{1}{6} s_{j}, v_{j}=\frac{5}{6} s_{j} .
$$

Once concluded this starting part of the investigation, on the basis of some findings (see Sect. 6), the indifference thresholds $\left\{q_{j}\right\}$ are also endogenously determined together with the weights $\left\{w_{j}\right\}$. Notice that the choice of this second set of parameters is mainly grounded on the fact that, for each indicator $I_{j}$, the indifference threshold constitutes a lower bound for the other thresholds, i.e. $q_{j} \leq p_{j} \leq v_{j}$. As far as the determination of the other parameters, i.e. $p_{j}$ and $v_{j}$, is concerned, we exogenously specify them again according to (7).

\subsection{The optimization problem}

In order to endogenously specify the above MURAME parameters through preference disaggregation, let us suppose that we have a reference set $A^{\prime}$ consisting of $m^{\prime}$ alternatives on which the classification is known a priori. The reference alternatives in this case, as it is often the case when the universe of alternatives $A$ is large, will be a subset $A^{\prime} \subset A$ of the whole set of the alternatives. In our implementation, $A^{\prime}$ will contain both a subset of nonbankrupt firms and a subset of bankrupt ones, for which we will assume that we know their a priori correct classification. Given this input, the objective is to determine the above parameters assigned to the MURAME criteria that will minimize the inconsistencies between the model classification of bankrupt firms and their correct one.

In order to formalize this optimization problem, initially with reference only to the weights $w_{j}$, suppose we are given a non-negative measure of inconsistency of our model $\mathcal{I}\left(w_{1}, \ldots, w_{n}\right)$, which ranges in the unit interval $[0,1]$, with $\mathcal{I}\left(\bar{w}_{1}, \ldots, \bar{w}_{n}\right)=$ 0 meaning that the weights $\bar{w}_{1}, \ldots, \bar{w}_{n}$ ensure the correct classification of the bankrupt firms. Then, our aim is to solve the following mathematical programming problem: 


$$
\begin{array}{rl}
\min _{w_{1}, \ldots, w_{n}} & \mathcal{I}\left(w_{1}, \ldots, w_{n}\right) \\
\text { s.t. } & w_{j} \geq 0, \quad j=1, \ldots, n \\
& \sum_{j=1}^{n} w_{j}=1 .
\end{array}
$$

Problem (8) has surely a very compact expression, but a more accurate analysis reveals possible serious difficulties when directly applying standard solvers for its solution. Indeed, in our case every evaluation of the function $\mathcal{I}\left(w_{1}, \ldots, w_{n}\right)$ first requires the computation of the final scores $\varphi\left(a_{i}\right)$, i.e. of (6), for each of the alternatives considered in the reference set, not only for the bankrupt ones. Then, it requires that the firms are classified according to (1), and finally, the measure of the inconsistency of the model can be computed. Observe that several problems arise when trying to handle formulation (8). Among them we highlight the following general issues, which may strongly affect the efficiency and effectiveness of solvers:

- It is quite hard to write an exact analytical expression in closed form for $\mathcal{I}\left(w_{1}, \ldots, w_{n}\right)$, in terms of the decision variables;

- The function $\mathcal{I}\left(w_{1}, \ldots, w_{n}\right)$ in general might be neither smooth nor Lipschitz/Holder continuous;

- The use of gradient-based methods for the solution of (8) is discouraged, since the derivatives of $\mathcal{I}\left(w_{1}, \ldots, w_{n}\right)$ are unavailable;

- An evolutionary optimizer to fast approximately solve (8) might be more appropriate, since expensive accurate solutions are possibly unnecessary.

Following this line of reasoning, in order to solve such a tough optimization problem, we use a solution algorithm based on PSO, which is a bio-inspired iterative metaheuristic for the solution of nonconvex global optimization problems (see Sect. 5 and Kennedy and Eberhart 1995; Blackwell et al. 2007).

Regarding the formulation in which both the weights $\left\{w_{j}\right\}$ and the indifference thresholds $\left\{q_{j}\right\}$ have to be endogenously determined, its structure substantially resembles (8). The only differences consist in the inclusion of the decision variables $q_{j}$ and of the constraints $q_{j} \geq 0$, with $j=1, \ldots, n^{4}$.

Lastly, concerning the specification of the measure of inconsistency $\mathcal{I}$ to use, we consider the following general principle: a good creditworthiness classification model should place as many bankrupt firms as possible in the worst rating class, and the number of bankrupt firms in each class should increase with the class, being the first one the best, and the last one the worst. By denoting with $n_{j}^{B}$ the number of bankrupt firms grouped by the model in $j$-th class and with $N^{B}$ the total number of bankrupt firms in our reference set, we considered then, according to the aforementioned principle, the following two measures of inconsistency:

\footnotetext{
${ }^{4}$ Notice that from conditions (7) one gets $q_{j}=p_{j} / 4$ for any $j$. So that, constraint $q_{j} \leq p_{j}$ (see Sect. 3) is satisfied by construction.
} 


$$
\begin{aligned}
& \mathcal{I}_{1}\left(w_{1}, \ldots, w_{n}\right) \doteq \mathcal{I}_{1}=\frac{n_{1}^{B}}{N^{B}} \\
& \mathcal{I}_{2}\left(w_{1}, \ldots, w_{n}\right) \doteq \mathcal{I}_{2}=1-\frac{n_{5}^{B}}{N^{B}} .
\end{aligned}
$$

We highlight that both these inconsistency measures are uniquely calculated with respect to the bankrupt firms in $A^{\prime}$.

The reasons for focusing only on the misclassification of bankrupt firms basically depend on the fact that the firms' credit status provided by the dataset used (see the next subsection) is a dichotomous variable, while the number of considered homogeneous rating classes is greater than two.

The choice to specialize our MURAME-based creditworthiness evaluation model in the bankrupt firms classification is grounded on the following awareness: while the wrong classification of a firm that will not bankrupt leads "only" to a missed earning for the involved creditor institution, the wrong classification of a firm that will bankrupt produces a certain loss for the same institution. As to say that in the former scenario, the economic-financial profile of the entailed institution remains unchanged, while in the latter scenario the same profile definitely worsens.

Clearly, an ideal approach would entail to jointly exploit the informative contents coming both from the bankrupt firms and from the nonbankrupt ones. In this regard, in Subsect. 7.2 we advance and apply a simple proposal about some inconsistency measures possibly capable to use the information deriving from both the categories of firms.

Notice that some additional hints for the classification of bankrupt firms might be possibly suggested by the use of standard classification measures like, for instance, the ROC analysis (see, e.g., Fawcett 2006; Hand and Till 2001; Waegeman et al. 2008).

\subsection{Balance sheet data on SMEs}

Our MURAME-based approach for creditworthiness evaluation, properly improved by the endogenous determination of some parameters, is applied to evaluate the creditworthiness of a set of Italian SMEs during the triennium 2006-2008. We highlight that, from the credit risk assessment standpoint, it is particularly important to be able to correctly classify bankrupt firms in a triennium like that, as it includes the beginning of the past economic and financial crisis.

Data has been collected from the professional database AIDA, by Bureau Van Dijk Electronic Publishing, which contains the balance sheets of Italian firms, drafted according to the IV CEE directive. The investigation regards SMEs with the legal status of companies or corporations, which have a good coverage in AIDA. Within the SMEs category, for the purpose of the present work, we focus on the enterprises having an annual turnover not exceeding 50 million Euros, and a number of employees between 50 and 249. Therefore, we consider the largest SMEs in terms of number of employees, which correspond to the third group of SMEs analyzed in Corazza et al. (2016).

As far as the choice of the balance sheet indicators is concerned, in order to be able to compare the results coming from this study with the ones obtained in Corazza et al. 
Table 1 Indicators considered in the creditworthiness analysis

\begin{tabular}{ll}
\hline$I_{1}$ & Cost of debt: Financial costs/Bank debts \\
$I_{2}$ & Return on equity (ROE): Net profit before tax/Total equity \\
$I_{3}$ & Total assets turnover: Sales/Total assets \\
$I_{4}$ & R\&D costs/Total asset \\
$I_{5}$ & Income tax/Profit before taxes \\
$I_{6}$ & Equity - Equipment \\
$I_{7}$ & Rate of increase of revenues from sales and services \\
$I_{8}$ & Liabilities/Total assets \\
$I_{9}$ & Cash/Total assets \\
$I_{10}$ & Working capital/Total assets \\
$I_{11}$ & Intangible/Total assets \\
$I_{12}$ & EBITDA/Total assets \\
$I_{13}$ & Retained earnings/Total assets \\
$I_{14}$ & Net income/Sales \\
$I_{15}$ & Short term debt/Equity \\
$I_{16}$ & EBITDA/Interest expenses \\
$I_{17}$ & Account payable/Sales \\
$I_{18}$ & Account receivable/Liabilities \\
$I_{19}$ & Sales/Personnel costs \\
\hline
\end{tabular}

Table 2 Cardinality of the sample of SMEs for each considered year

\begin{tabular}{llll}
\hline Years & $\begin{array}{l}\text { Active } \\
\text { firms }\end{array}$ & $\begin{array}{l}\text { Bankrupt } \\
\text { firms }\end{array}$ & Total \\
\hline 2006 & 6625 & 1089 & 7714 \\
2007 & 6766 & 925 & 7691 \\
2008 & 6933 & 696 & 7629 \\
\hline
\end{tabular}

(2016), we considered the same two sets of evaluation criteria used in that paper. The first set is constituted by 19 quantitative indicators frequently used in the literature to measure profitability, liquidity, solvency and other aspects of firm's profile (see Table 1). Once specified the first set of evaluation criteria, it is possible to detect in the database AIDA the firms for which such indicators are available. In Table 2, we report the cardinality of the sample of SMEs which are present in AIDA for at least two years of the considered triennium; the table illustrates separately the number of active and bankrupt firms analyzed. The second set of evaluation criteria, which is a subset of the first one, is constituted by the accounting indicators used in Altman and Sabato (2007) for the prediction of U.S. SMEs default (in the sequel named Altman's variables). Altman's variables are listed in Table 3 and correspond to the indexes $I_{15}$, $I_{9}, I_{12}, I_{13}$ and $I_{16}$ of Table 1 .

The use of two different sets of evaluation indicators allows us to clearly exemplify the impact of the parameter specification on the scoring and on the ranking of the firms, as well as on their classification into homogeneous rating classes. In particular, 
Table 3 Altman's variables in the US SME model of Altman and Sabato (2007)

\begin{tabular}{ll}
\hline Accounting ratio category & Variable \\
\hline Leverage & Short term debt/Equity book value \\
Liquidity & Cash/Total assets \\
Profitability & EBITDA/Total assets \\
Coverage & Retained earnings/Total assets \\
Activity & EBITDA/Interest expenses \\
\hline
\end{tabular}

all the applications in Corazza et al. (2016) were performed under an equally weighting scheme, i.e. all the values of the weights were equal to $1 / 19$ for the indicators belonging to the first set and to $1 / 5$ for the Altman's variables. Of course, from a technical point of view, moving from the former indicators to the Altman's variables is equivalent to a change of the preference structure implicit in the model, in terms of importance assigned to the indicators. Indeed, in such a case the value of the weight of each Altman's variable becomes $1 / 5$, and the value of the weight of any other variable becomes 0 . Furthermore, the Altman's variables represent approximately $25 \%$ of all the variables in Table 1, as to say that the informative contents of the two sets of variables, in Table 1 and Table 3, respectively, are so different that a significant impact on the rating is expected.

The results in terms of distribution of bankrupt firms in rating classes presented in Corazza et al. (2016, Tables 15, 32, 33) show that, in all the considered years, there is a slight decrease of the number of bankrupt firms in the first (best) class, and a slight increase of the number of the bankrupt enterprises in the last (worst) class. Therefore, it appears that MURAME methodology, when using only the Altman's variables, rates slightly better the bankrupt firms in the extreme classes than when using all the variables in Table 1.

This remark suggests another reason for the (initial) choice of the weights $\left\{w_{j}\right\}$ as parameters to endogenously determine. Indeed, a specific tuning of them might be a possible critical issue in order to improve the model performance.

\section{Using PSO evolutionary method to solve the optimization problem} (8)

Broadly speaking, the basic idea behind PSO is to use the so called swarm intelligence (see Bonabeau et al. 1999), that drives groups of individuals belonging to the same species when foraging. Every member of the swarm explores the search area keeping memory of its best position so far outreached, and it exchanges this information with the individuals belonging to a suitable neighborhood in the swarm. Thus, the whole swarm is supposed to converge eventually to the best global position reached by some swarm members. From a mathematical point of view, every member of the swarm (namely a particle) represents a possible solution point in the feasible set of the investigated optimization problem. Moreover, every particle is randomly initialized before PSO 
procedure is applied and it is also associated with a vector of velocity, also randomly initialized, which is used to determine its direction of movement.

Now, in order to formalize these ideas, let us denote with $P$ the size of the swarm, and let $f: \mathbb{R}^{n} \mapsto \mathbb{R}$ be the function to minimize. For each particle $l=1, \ldots, P$, let $\mathbf{x}_{l}^{k} \in \mathbb{R}^{n}$ be its position at step $k=0,1, \ldots$ of the PSO procedure. Then, its new position at step $k+1$ is

$$
\mathbf{x}_{l}^{k+1}=\mathbf{x}_{l}^{k}+\mathbf{v}_{l}^{k+1}, \quad l=1, \ldots, P,
$$

where $\mathbf{v}_{l}^{k+1}$ is an $n$-real vector which is usually addressed in the literature as velocity, and is given by

$$
\mathbf{v}_{l}^{k+1}=\chi^{k}\left[w^{k} \mathbf{v}_{l}^{k}+\boldsymbol{\alpha}_{l}^{k} \otimes\left(\mathbf{p}_{l}^{k}-\mathbf{x}_{l}^{k}\right)+\boldsymbol{\beta}_{l}^{k} \otimes\left(\mathbf{p}_{g}^{k}-\mathbf{x}_{l}^{k}\right)\right]
$$

In (11) $\chi^{k}>0$ is a suitable parameter, $\mathbf{v}_{l}^{k}$ is the previous vector of velocity, and $\mathbf{p}_{l}^{k}$ and $\mathbf{p}_{g}^{k}$ are, respectively, the best solution so far found by particle $l$ and by the whole swarm, i.e.

$$
\begin{aligned}
& \mathbf{p}_{l}^{k} \in \arg \min _{0 \leq h \leq k}\left\{f\left(\mathbf{x}_{l}^{h}\right)\right\}, \quad l=1, \ldots, P, \\
& \mathbf{p}_{g}^{k} \in \arg \min _{1 \leq l \leq P}\left\{f\left(\mathbf{p}_{l}^{k}\right)\right\} .
\end{aligned}
$$

Finally, $\boldsymbol{\alpha}_{l}^{k}, \boldsymbol{\beta}_{l}^{k} \in \mathbb{R}^{n}$ are positive random vectors, with the symbol $\otimes$ denoting the component-wise product. The most commonly used specifications in the literature for $\boldsymbol{\alpha}_{l}^{k}, \boldsymbol{\beta}_{l}^{k}$, which we will adhere to, are (see Blackwell et al. 2007)

$$
\begin{aligned}
\boldsymbol{\alpha}_{l}^{k} & =c_{1} \mathbf{r}_{l, 1}^{k}, \\
\boldsymbol{\beta}_{l}^{k} & =c_{2} \mathbf{r}_{l, 2}^{k},
\end{aligned}
$$

where $\mathbf{r}_{l, 1}^{k}, \mathbf{r}_{l, 2}^{k}$ are $n$-real vectors whose entries are uniformly randomly distributed in $[0,1]$, and $c_{1}, c_{2} \in(0,2.5]$. A full discussion on the choice of the parameters in (11) can be found in Serani et al. (2016).

In the remainder of this section, we present our original enhancements to PSO, in order to improve its effectiveness when tackling the optimization problem. Initially, in order to manage the constraints of (8), we replace the penalty function approach proposed and used in Corazza et al. (2015b) with a nonlinear reformulation of the optimization problem. This allows us to avoid possible numerical ill-conditioning when assessing the penalty parameters. Then, with reference to the initialization of the particles, we endow PSO with some recently introduced initialization procedures never used before in this research area. 


\subsection{A nonlinear reformulation of the optimization problem (8)}

Since PSO was conceived for unconstrained problems, its direct application to (8) cannot prevent from generating infeasible particles' positions when constraints are considered. To avoid this problem, different strategies have been proposed in the literature, and most of them involve repositioning of the particles (see for instance Zhang et al. 2005), or the introduction of some external criteria to rearrange the components of the particles (see for instance Cura 2009), or the use of a penalty function approach (see for instance Corazza et al. 2013, 2015b). On the contrary, in this paper we consider a novel approach which first encompasses a nonlinear reformulation of (8), so that we can maintain PSO as in its original iteration. Initially, with reference to the endogenous determination of only the weights $\left\{w_{j}\right\}$, in place of (8) we can solve the unconstrained optimization problem

$$
\min _{t_{1}, \ldots, t_{n}} \mathcal{I}\left[w_{1}(\mathbf{t}), \ldots, w_{n}(\mathbf{t})\right]
$$

where the mapping between the new variables, i.e. $\left(t_{1}, \ldots, t_{n}\right)$, and the old ones, i.e. $\left(w_{1}, \ldots, w_{n}\right)$, is given by the following nonlinear transformation

$$
w_{j}(\mathbf{t}) \leftarrow \frac{\psi_{j}(\mathbf{t})}{\sum_{i=1}^{n} \psi_{i}(\mathbf{t})}, \quad j=1, \ldots, n
$$

where we assume $\psi_{j}(\mathbf{t})$ continuous and $\psi_{j}(\mathbf{t}) \geq 0$. Observe that any global solution $\overline{\mathbf{t}} \in \mathbb{R}^{n}$ of (16) corresponds to a global solution $\overline{\mathbf{w}} \leftarrow \mathbf{w}(\overline{\mathbf{t}})$ of (8), where $\mathbf{w}(\mathbf{t})=\left(w_{1}(\mathbf{t}), \ldots, w_{n}(\mathbf{t})\right)^{T}$, even though (17) surely introduces nonlinearities which in principle increase the computational complexity. Apart from the last drawback, we remark that in case the vector $\overline{\mathbf{t}} \neq 0$ solves (16), by (17) the vector $\mathbf{w}(\overline{\mathbf{t}})$ is a feasible point for (8).

Considering that on the overall we are adopting a metaheuristic procedure to approximately solve (8), the following two simple choices may be considered, with some care, for $\psi_{j}(\mathbf{t})$ in our framework, namely

$$
\begin{aligned}
& \psi_{j}(\mathbf{t})=t_{j}^{2}, \quad j=1, \ldots, n, \\
& \psi_{j}(\mathbf{t})=\left|t_{j}\right|, \quad j=1, \ldots, n .
\end{aligned}
$$

In particular, we adopted (18) in our numerical experience (but also (19) might have been an appealing choice). The transformation (18) is continuous everywhere, excluding a neighborhood of $\mathbf{t}=0$, and $\mathbf{t}=0$ cannot correspond to any feasible set of weights in (8). Thus, recalling that PSO is a metaheuristics which does not guarantee any convergence to a global minimum, the use of (18) to large extent can be seen as a reasonable expedient to transform the constrained problem (8) into the unconstrained one (16), without a significant additional computational burden. We remark that in our implementation of PSO for solving (16), we introduced a check to prevent from 
possible division by small values in (17), when (18) is adopted. Anyway, in our numerical experience the latter test was never satisfied, since the algorithm likely tended to approach a global minimum and skip the point $\mathbf{t}=0$.

As concerns the instance in which both weights and indifference thresholds have to be endogenously determined, similarly to (17), to prevent the possible generation of infeasible values for the decision variables $\left\{q_{j}\right\}$, we adopted the quadratic transformation $q_{j} \leftarrow u_{j}^{2}$, with $j=1, \ldots, n$, and we solved by PSO an unconstrained optimization problem analogous to (16), for any of the two objective functions (9).

\subsection{The penalty function approach, for comparison purposes}

The (novel) unconstrained reformulation (16) of problem (8), which allows to keep PSO as in its original iteration, is not the only possible one. Indeed, as already mentioned, a penalty function approach is proposed and used in Corazza et al. (2013, 2015 b) for reformulating the mathematical programming problems therein involved. In this subsection we synthetically present this latter approach; the results of its application are presented for comparison purposes in Subsect. 7.1.

Roughly speaking, in case of minimization as in (8), the idea behind the penalty function approach is to reformulate the starting constrained optimization problem in a new unconstrained one, in which the new objective function is given by adding a properly penalized sum of all the constraints violations to the original objective function. With particular reference to (8), the application of this approach leads to the following unconstrained optimization problem

$$
\min _{w_{1}, \ldots, w_{n}} \mathcal{I}\left(w_{1}, \ldots, w_{n}\right)+\frac{1}{\epsilon}\left(\sum_{j=1}^{n} \max \left\{0,-w_{j}\right\}+\left|\sum_{j=1}^{n} w_{j}-1\right|\right)
$$

where $\epsilon$ is the so-called penalty parameter. It is possible to prove that there exists a penalty parameter value $\epsilon^{*}$ such that, under mild assumptions, for any $\epsilon \in\left(0, \epsilon^{*}\right)$ the solutions of (20) and of the constrained problem (8) coincide (see for details Corazza et al. 2013, 2015b and the references therein).

As regards the instance in which both weights $\left\{w_{j}\right\}$ and indifference thresholds $\left\{q_{j}\right\}$ have to be endogenously determined, the structure of the new objective function remains basically the same. The only difference consists of including constraints violations associated to $q_{j} \geq 0$, with $j=1, \ldots, n$.

Notice that, by construction, the penalty function approach suffers from some drawbacks which, on the contrary, are not present in the nonlinear reformulation approach proposed and used in this paper. In particular we have the following:

- The proposal in Subsect. 5.1 always ensures that the found (sub)-optimal solution of problem (8) is always feasible; on the contrary, the last property does not necessarily hold for the penalty function-based approach;

- The approach in Subsect. 5.1 does not require the burdensome assessment of the penalty parameter $\epsilon$; 
- As a well known drawback from the literature (see, e.g., Corazza et al. 2013), in the penalty function-based approach the use of small values of the penalty parameter $\epsilon$ may likely yield ill-conditioning; conversely, large values of $\epsilon$ might not be satisfactory in order to guarantee the feasibility for the found solution.

\subsection{The initialization procedures}

As for every evolutionary algorithm, PSO performance depends on the choice of its parameters $\chi, w, c_{1}, c_{2}$ and on the initial positions and velocities of the swarm, that is $\mathbf{x}_{l}^{0}, \mathbf{v}_{l}^{0} \in \mathbb{R}^{n}$ for $l=1, \ldots, P$. For the choice of the parameters, we complied with standard settings in the literature. As regards the initialization of the algorithm, we applied and compared three different proposals: the standard random one, mainly adopted in the literature, and two deterministic ones, namely Orthoinit and Orthoinit+, recently proposed in Corazza et al. (2015a) and Diez et al. (2016), respectively. The idea behind these two novel initializations is to scatter particle trajectories in the search space in the early iterations, in order to better initially explore the search space, and to obtain approximate solutions that are not grouped in a reduced sub-region of the feasible set. In the Appendix, we detail a brief summary of the theoretical results supporting these initializations (see Diez et al. 2016 for a more complete report).

Assuming for simplicity $P=2 n$, recalling the formulae (29), if we adopt the following initialization (Orthoinit) for PSO particles

$$
\left(\begin{array}{c}
\mathbf{v}_{i}^{0} \\
\mathbf{x}_{i}^{0}
\end{array}\right)=\rho_{i} z_{i}(k), \quad \rho_{i} \in \mathbb{R} \backslash\{0\}, \quad i=1, \ldots, n,
$$

and

$$
\left(\begin{array}{c}
\mathbf{v}_{n+i}^{0} \\
\mathbf{x}_{n+i}^{0}
\end{array}\right)=\rho_{n+i} z_{n+i}(k), \quad \rho_{n+i} \in \mathbb{R} \backslash\{0\}, \quad i=1, \ldots, n,
$$

then the first $n$ entries of the free responses of the particles, i.e. the velocities $\mathbf{v}_{i}^{0}$, $i=1, \ldots, 2 n$, are orthogonal at step $k$ of the deterministic PSO. To some extent, this also tends to impose a near orthogonality of the particles trajectories at step $k$, as well as in some subsequent iterations. While this initialization has the advantage of making the particles trajectories better scattered in the search space, unfortunately it tends to yield too sparse approximate solutions (see Diez et al. 2016), i.e. only few components of the vector of solutions are nonzero. This is essentially a consequence of the fact that $z_{i}(k)$ and $z_{n+i}(k)$ are very sparse vectors (see the Appendix).

In order to possibly pursue a dense final solution, a modification (namely Orthoinit+) has been proposed in Diez et al. (2016). Here, the vectors $z_{i}(k)$, $i=1, \ldots, 2 n$ in (21) and (22) are replaced by the following ones 


$$
\begin{gathered}
v_{i}(k)=z_{i}(k)-\alpha \sum_{\substack{j=1 \\
j \neq i}}^{n} z_{j}(k)-\gamma \sum_{j=n+1}^{2 n} z_{j}(k), \quad i=1, \ldots, n \\
v_{n+i}(k)=z_{n+i}(k)-\beta \sum_{\substack{j=n+1 \\
j \neq n+i}}^{2 n} z_{j}(k)-\delta \sum_{j=1}^{n} z_{j}(k), \quad i=1, \ldots, n,
\end{gathered}
$$

where $\alpha \in \mathbb{R} \backslash\left\{-1, \frac{1}{n}\right\}, \quad \beta=\frac{2}{n-2}, \quad \gamma=0, \quad \delta \in \mathbb{R} \backslash\{0,1\}$. It is possible to prove that the vectors $v_{1}(k), \ldots, v_{2 n}(k)$ are still well scattered in $\mathbb{R}^{2 n}$, as well as uniformly linearly independent (see Diez et al. 2016).

\section{Numerical results}

The ideal application of the preference disaggregation methodology described above would imply to consider the whole group of firms, that is to set $A^{\prime} \equiv A$, and then to determine the weights (or the weights and the indifference thresholds) that minimize the measure of inconsistency $\mathcal{I}(\cdot, \ldots, \cdot)$ over the entire population of firms, for each of the considered years. On the basis of some preliminary trials, a single evaluation of $\mathcal{I}(\cdot, \ldots, \cdot)$ requires on average 16 minutes on an Intel ${ }^{\mathrm{TM}}$ i7-based PC with $16 \mathrm{~GB}$ RAM using the coding environment Matlab ${ }^{\complement}$, release 2016b. This means that for a single run of our PSO-based solver with $P=40$ particles, around 11 hours are needed ${ }^{5}$. To tackle this drawback, on the basis of the results obtained in Corazza et al. (2015b), we considered a smaller reference set of firms in order to achieve a good compromise between the quality of the obtained solution and the computational time required. As a matter of fact, the larger the cardinality of $A^{\prime}$, the better the classification performance for the group of considered firms, but also the longer the computational time. Furthermore, again with respect to the time of computation, we want also to check if, similarly to Corazza et al. (2015a), the use in these experiments of PSO initializations Orthoinit and Orthoinit+ allows a better minimization of $\mathcal{I}(\cdot, \ldots, \cdot)$ within the early iterations, compared to the one obtained with the usual random initialization of the PSO particles. This fact, if verified, could be useful to quickly finding approximate solutions when the size of $A^{\prime}$ is particularly large, and consequently, the time needed for each iteration of the PSO-based solver is quite long.

Regarding the setting of the PSO parameters used in our experiments, they have been determined according to the prevailing specialized literature and analogously to Corazza et al. (2015a), that is: $c_{1}=c_{2}=1.49618, w^{k}=w=0.7298, \chi^{k}=\chi=1$ for all $k, \alpha=0.25$ and $\delta=0.75$.

Then, to keep our preference disaggregation procedure safe and significant: first, the members of the reference set $A^{\prime}$ are randomly selected from the whole population of the considered firms, i.e. $A$, preserving the same ratio between active and bankrupt firms as in $A$ itself. Then, the selected optimization approach is applied to $A^{\prime}$ in order

\footnotetext{
5 This time might be reduced by the parallelization of the code used for the computations, but such an issue is not treated in this paper.
} 
Table 4 Average $(\mu)$ and standard deviation $(\sigma)$ values of $\mathcal{I}_{1}$ for differently sized reference sets $\left(A^{\prime}\right)$ and for the whole population of firms $(A)$

\begin{tabular}{llll}
\hline & Random & Orthoinit & Orthoinit+ \\
\hline$\left|A^{\prime}\right|=250$ & & & \\
$\mu\left(A^{\prime}\right)$ & 0.534783 & 0.495652 & 0.547826 \\
$\sigma\left(A^{\prime}\right)$ & 0.055168 & 0.070644 & 0.078261 \\
$\mu(A)$ & 0.681466 & 0.643247 & 0.626437 \\
$\sigma(A)$ & 0.048448 & 0.047240 & 0.022899 \\
\hline$A^{\prime} \mid=2500$ & & & \\
$\mu\left(A^{\prime}\right)$ & 0.580263 & 0.522807 & 0.587719 \\
$\sigma\left(A^{\prime}\right)$ & 0.015451 & 0.042920 & 0.015445 \\
$\mu(A)$ & 0.608046 & 0.594109 & 0.603879 \\
$\sigma(A)$ & 0.011598 & 0.019901 & 0.004454 \\
\hline
\end{tabular}

to compute weights and possibly indifference thresholds; lastly, these parameters are used to classify all firms belonging to $A$.

Finally, as for an appropriate determination of the cardinality of $A^{\prime}$, after some preliminary tests on the sensitivity of the inconsistency measures with respect to the cardinality itself, we set the value $\left|A^{\prime}\right|=2500$. This allows to perform nearly 500 iterations of the PSO-based solver within a reasonable CPU-time. In order to give an example for illustrating the rationale under our choice of the cardinality of $A^{\prime}$, in Table 4 we provide some sample statistics computed over the values of the inconsistency measure $\mathcal{I}_{1}$, obtained after ten runs of the PSO-based solver, using all the indicators of Table 1. In particular, in each run a new randomly selected $A^{\prime}$ is considered. It can be seen that the average (sub-)optimal value of $\mathcal{I}_{1}$ computed using a smaller reference set (i.e. $\left|A^{\prime}\right|=250$ ) is better than that for a larger one (i.e. $\left|A^{\prime}\right|=2500$ ), while the converse holds for the results computed over the whole population of firms (i.e. A). Notice that these findings hold regardless of the initialization procedure considered, and similar conclusions hold for the inconsistency measure $\mathcal{I}_{2}$, too.

\subsection{Results: the creditworthiness classifications with decision variables w}

In Tables 5, 6, 7 and 8, we show the results, in terms of classification both of the bankrupt firms and of the entire population of the investigated Italian SMEs firms, using data of year $2008^{6}$. The reported percentages represent the distribution of the firms in the five rating classes obtained using the weights $\left\{w_{j}(\mathbf{t})\right\}$ as specified in (17) and (18), adopting the two inconsistency measures $\mathcal{I}_{1}$ and $\mathcal{I}_{2}$, and employing the three PSO initialization procedures described in Subsect. 5.3 (see the rows labeled "Random," "Orthoinit" and "Orthoinit+"). In particular, Tables 5 and 6 provide the results considering all the indicators of Table 1, while Tables 7 and 8 consider only the Altman's variables of Table 3 . We remind that the values of the weights $\left\{w_{j}(\mathbf{t})\right\}$

\footnotetext{
${ }^{6}$ Notice that we performed all the experiments described in this section for any year of the considered triennium, (i.e., 2006-2008). The results coming from each of the various years are definitely similar, so we report only those of 2008 .
} 
have been detected applying the solution algorithm to a reference set of firms $A^{\prime}$ of size 2500, and that the classification results reported here refer to the application of MURAME once set with the obtained weights to the entire population of firms. Lastly, for comparison purposes, in the first row of each table (labeled "Standard") we give the classification results got using the standard exogenous specification of weights adopted in Corazza et al. (2016), that is $w_{j}=1 / n$ for the weights and $q_{j}=s_{j} / 6$ for the indifference thresholds, with $j=1, \ldots, n$.

From the results presented in these tables, it can be seen that:

- When considering as criteria either all the indicators of Table 1 or only the Altman's variables and using either the inconsistency measure $\mathcal{I}_{1}$ or the inconsistency measure $\mathcal{I}_{2}$, the (sub-)optimal values of the weights $\left\{w_{j}(\mathbf{t})\right\}$ determined by our PSO-based solver allow improvements in terms of the creditworthiness distribution of bankrupt firms with respect to the exogenous specification of the same weights (see Tables 5 and 6);

- The overall quality of the creditworthiness distribution of bankrupt firms depends on the PSO initialization procedure applied. Indeed, in the case of the Orthoinit one, when all the nineteen indicators of Table 1 are considered and either using $\mathcal{I}_{1}$ or $\mathcal{I}_{2}$, the obtained classification (slightly) dissatisfies the aforementioned general principle, since it shows an higher concentration of bankrupt firms in the fourth rating class (see Tables 5 and 6);

- Conversely, when considering only the five Altman's variables, some little improvements in the bankrupt firms' creditworthiness classification are observed, generally regardless of the initialization procedure or the inconsistency measure adopted, with respect to the results obtained when using all the 19 indicators of Table 1 (see Tables 7 and 8).

\subsection{Results: the creditworthiness classifications with decision variables $w$ and $q$}

Now, we present the classification results related to the endogenous determination of both the weights and the indifference thresholds together. In other terms, we add to our optimization framework the decision variables $q_{j}$, with $j=1, \ldots, n$. In this case, we focus only on the Altman's variables since, as observed in the previous subsection, their use leads to some improvements in the classification results with respect to the use of all the indicators of Table 1 . Tables 9 and 10 show the classification results got when using $\mathcal{I}_{1}$ and $\mathcal{I}_{2}$, respectively.

In this case, it can be noted that:

- In general, good results are obtained in terms of the percentages of bankrupt firms classified in the best creditworthiness class, either using the inconsistency measure $\mathcal{I}_{1}$ or the inconsistency measure $\mathcal{I}_{2}$ and regardless of the initialization procedure (see Tables 9 and 10, respectively). Moreover, these percentages are all better than the corresponding ones reported in Tables 7 and 8, respectively;

- Nevertheless, when using $\mathcal{I}_{1}$ and independently of the initialization procedure employed, the creditworthiness classifications obtained always fail to satisfy the general principle considered in Sect. 4.1; 


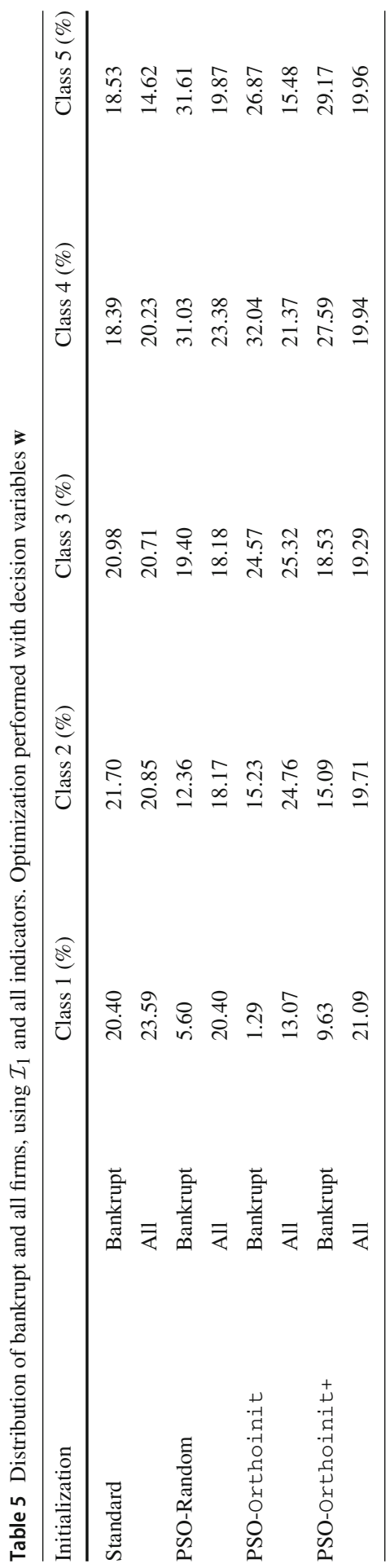




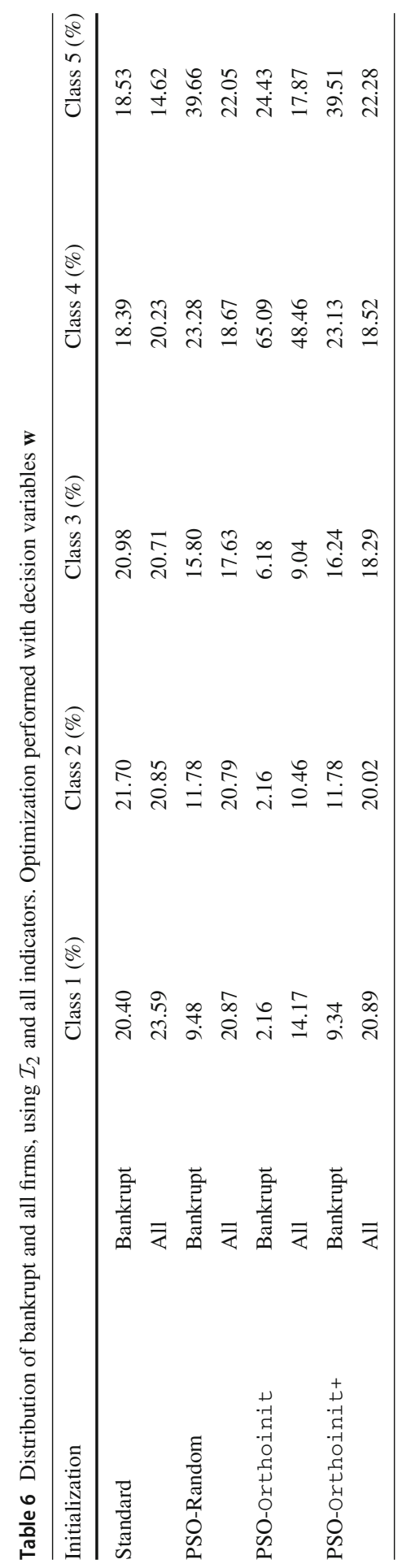




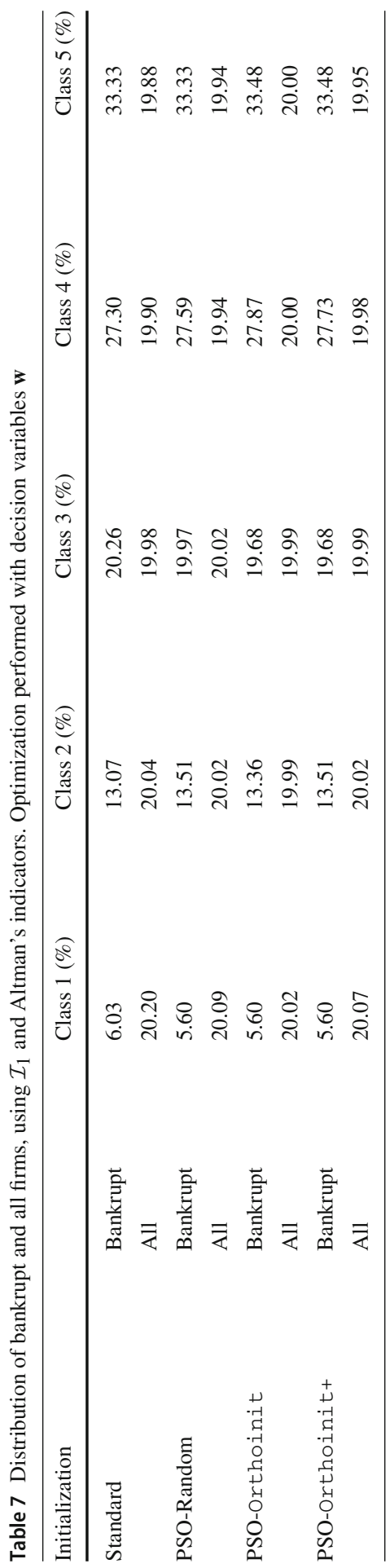




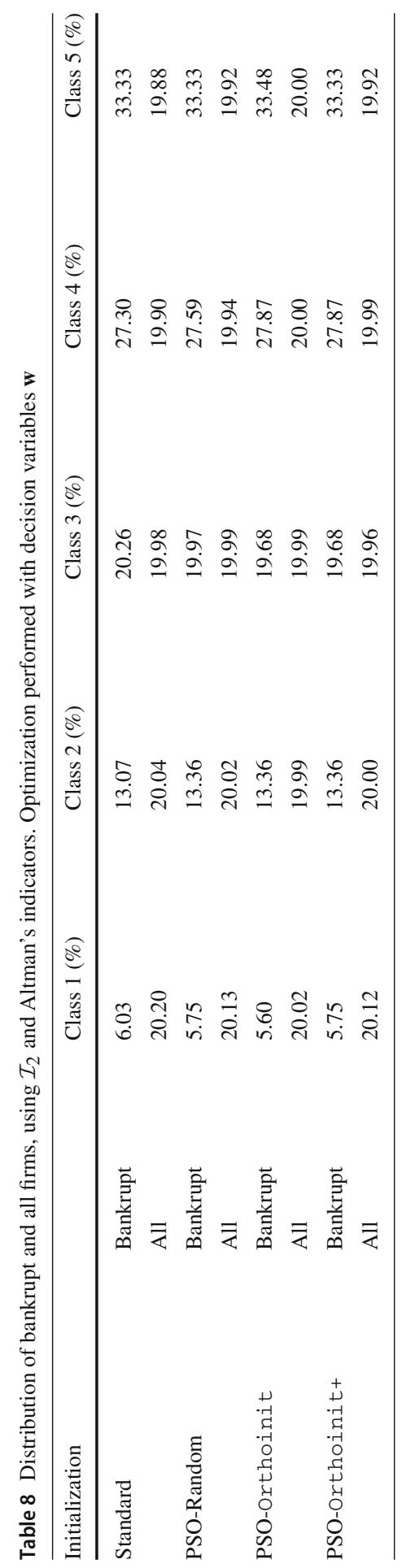




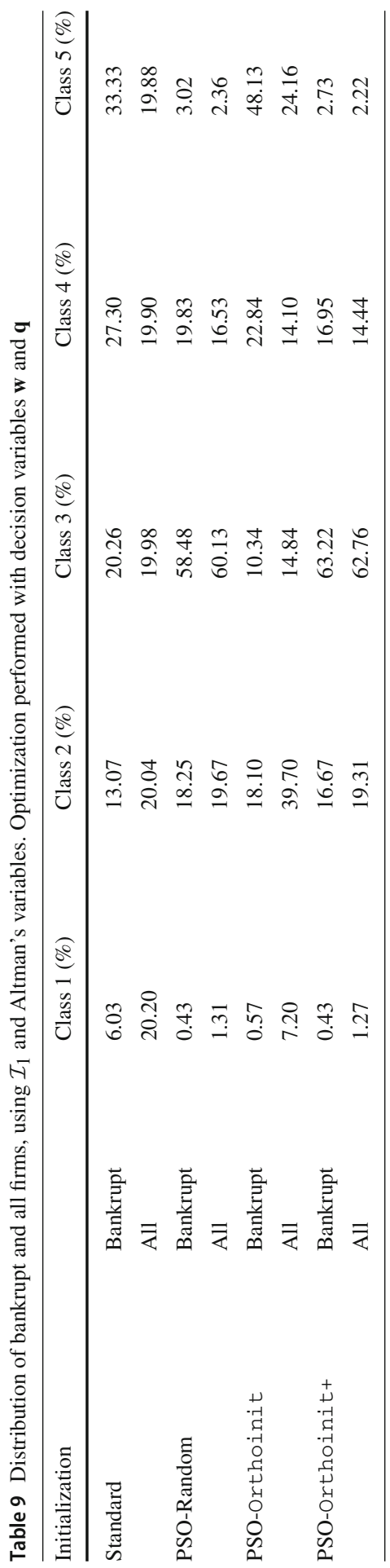




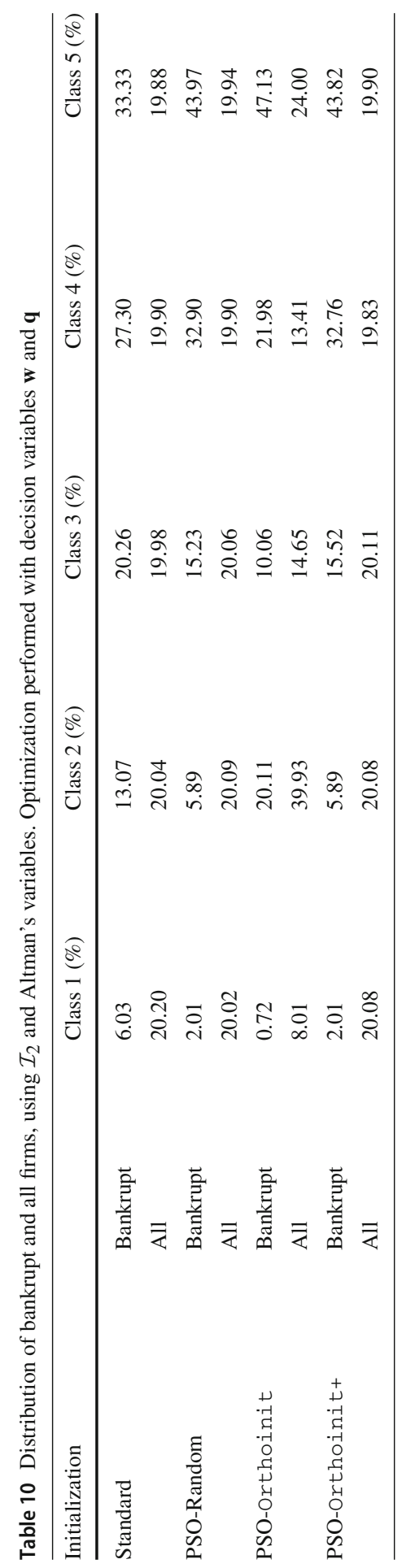


- Conversely, when using $\mathcal{I}_{2}$, the same general principle as above is dissatisfied only once, in correspondence of the initialization procedure Orthoinit (see Table 10). Furthermore, again when using $\mathcal{I}_{2}$ and regardless of the initialization procedure, remarkable improvements are achieved in terms of the percentages of bankrupt firms classified in the worst creditworthiness class, with respect to the corresponding ones presented in Table 8.

At this point of our analysis, we can draw a first general conclusion. The results reported in Tables 5, 6, 7, 8, 9 and 10 show with some evidence that the best creditworthiness distributions of bankrupt firms are obtained considering $\mathcal{I}_{2}$ as inconsistency measure. Furthermore, they also show that, albeit with less evidence, some of the obtained creditworthiness classifications yield improvements when jointly using $\mathbf{w}$ and $\mathbf{q}$ as decision variables.

\subsection{Results: the endogenously determined MURAME parameters}

Here, we provide some observations about the (sub-)optimal values of the MURAME parameters endogenously determined using the inconsistency measure $\mathcal{I}_{2}{ }^{7}$. These values are presented in Tables 11 and 12 . In particular: Table 11 refers to the case with all indicators of Table 1 and only the weights $\mathbf{w}$ as decision variables; Table 12 refers to the case with only the Altman's variables of Table 3 with both the weights $\mathbf{w}$ and the indifference thresholds $\mathbf{q}$ as decision variables.

From the displayed values, it can be seen that:

- A drawback related to the use of the initialization procedure Or thoinit emerges, as already highlighted in Corazza et al. (2015a): namely, the sparsity of the achieved solutions ${ }^{8}$. Indeed, while both the other two initialization procedures give dense solutions ${ }^{9}$, those provided by Or thoinit lead to a creditworthiness classification model in which just one or at most two criteria play some role;

- Then, from Table 11 one can infer that a careless initialization of PSO, like that performed by the initialization procedure Random, may be unable to lead to clear indications about the most relevant criteria. Indeed, using Random a too large number of criteria is suggested, which does not help to rank them by their importance. This motivates the introduction of the initialization procedure Orthoinit+, whose performance generally constitutes a balance between the extreme ones provided by Random and Orthoinit.

- Finally, again with reference to Table 11, one can note that the PSO-based solver initialized through Orthoinit + identifies $I_{2}, I_{3}$ and $I_{5}$ as relevant criteria, while assigns the value $0.06 \%$ to the other weights, suggesting the scarce relevance of the latter. We highlight that, even if the obtained values for the endogenously determined MURAME parameters may appear unusual in a creditworthiness evaluation model, two aspects have to be remarked. First aspect: such values represent a (sub)optimal solution determined by a metaheuristic. In general, ceteris paribus, the

\footnotetext{
7 The choice of considering only $\mathcal{I}_{2}$ is based on the previous general conclusion.

8 For the sake of brevity, hereafter by "solution" we mean "(sub-)optimal solution."

9 By "dense solution" we mean that the majority of the weights, or of the weights and the indifference thresholds, have significant non-zero values.
} 
Table 11 Values of the weights of all indicators using $\mathcal{I}_{2}$

\begin{tabular}{|c|c|c|c|c|c|}
\hline Initialization & $I_{1}(\%)$ & $I_{2}(\%)$ & $I_{3}(\%)$ & $I_{4}(\%)$ & $I_{5}(\%)$ \\
\hline PSO-Random & 0.01 & 34.22 & 14.76 & 0.01 & 11.56 \\
\hline PSO-Orthoinit & 0.00 & 0.00 & 0.00 & 0.00 & 0.00 \\
\hline \multirow[t]{2}{*}{ PSO-Orthoinit+ } & 0.06 & 57.17 & 38.41 & 0.06 & 3.45 \\
\hline & $I_{6}(\%)$ & $I_{7}(\%)$ & $I_{8}(\%)$ & $I_{9}(\%)$ & $I_{10}(\%)$ \\
\hline PSO-Random & 0.00 & 4.84 & 2.50 & 10.92 & 4.37 \\
\hline PSO-Orthoinit & 0.00 & 0.00 & 0.00 & 0.00 & 0.00 \\
\hline \multirow[t]{2}{*}{ PSO-Orthoinit+ } & 0.06 & 0.06 & 0.06 & 0.06 & 0.06 \\
\hline & $I_{11}(\%)$ & $I_{12}(\%)$ & $I_{13}(\%)$ & $I_{14}(\%)$ & $I_{15}(\%)$ \\
\hline PSO-Random & 0.02 & 0.03 & 3.27 & 0.59 & 3.34 \\
\hline PSO-Orthoinit & 0.00 & 0.00 & 0.00 & 0.00 & 0.00 \\
\hline \multirow[t]{2}{*}{ PSO-Orthoinit+ } & 0.06 & 0.06 & 0.06 & 0.06 & 0.06 \\
\hline & $I_{16}(\%)$ & $I_{17}(\%)$ & $I_{18}(\%)$ & $I_{19}(\%)$ & \\
\hline PSO-Random & 2.75 & 4.99 & 0.41 & 1.40 & \\
\hline PSO-Orthoinit & 100.00 & 0.00 & 0.00 & 0.00 & \\
\hline PSO-Orthoinit+ & 0.06 & 0.06 & 0.06 & 0.06 & \\
\hline
\end{tabular}

Table 12 Values of the weights and the indifference thresholds of the Altman's variables using $\mathcal{I}_{2}$

\begin{tabular}{|c|c|c|c|c|c|c|}
\hline Initialization & & $I_{9}$ & $I_{12}$ & $I_{13}$ & $I_{15}$ & $I_{16}$ \\
\hline \multirow[t]{2}{*}{ PSO-Random } & $\mathbf{w}$ & $0.92 \%$ & $56.54 \%$ & $15.33 \%$ & $1.28 \%$ & $25.93 \%$ \\
\hline & $\mathbf{q}$ & 6.994 & 5.468 & 2.886 & 20.714 & 0.021 \\
\hline \multirow[t]{2}{*}{ PSO-Orthoinit } & $\mathbf{w}$ & $0.00 \%$ & $0.00 \%$ & $50.53 \%$ & $0.00 \%$ & $49.47 \%$ \\
\hline & $\mathbf{q}$ & 0.000 & 0.000 & 0.000 & 0.000 & 1.549 \\
\hline \multirow[t]{2}{*}{ PSO-Orthoinit+ } & $\mathbf{w}$ & $13.97 \%$ & $21.51 \%$ & $21.51 \%$ & $21.51 \%$ & $21.51 \%$ \\
\hline & $\mathbf{q}$ & 0.161 & 0.161 & 0.161 & 22.829 & 0.161 \\
\hline
\end{tabular}

solution performances of a metaheuristic worsen when the dimension of the search space increases, as in the considered case. Therefore, as per operational practice, in order to improve the quality of the solution it might be necessary to increase the number of PSO iterations. Of course, this would have negative consequences in terms of the computational time required. Second aspect: we highlight that the determined values of the MURAME parameters are however significantly informative. Indeed, recalling that the main aim of a MCDA-based model is to support the DM in the decision process, the obtained values of the weights can represent a tentative assessment for a better understanding of the relevance of the various criteria. Similarly, the achieved values of the indifference thresholds can represent a basis for a better understanding of the preference structure implicit in the used data. 
At the end of this subsection, we can draw another general conclusion, from the results reported in Tables 5, 6, 7, 8, 9, 10, 11 and 12. Namely, in terms of both creditworthiness classification and tuning of MURAME parameters, Orthoinit+ initialization seems preferable. This can be explained by noting that Orthoinit+ adopts an initial population which proves to be more scattered on the search space, with respect to both Orthoinit and Random initializations. Thus, better approximate solutions can be obtained by PSO scheme, inasmuch as a better exploration of the search space is expected.

\subsection{Some in-depth-analyses about the deterministic initialization procedures}

As mentioned before, we want to check the capability of the two deterministic PSO initialization procedures in order to find reasonably good solutions in the early iterations of PSO-based solver. This issue could be of interest in case our creditworthiness evaluation model was applied to a reference set $A^{\prime}$ whose cardinality is particularly large.

As examples, Figs. 1 and 2 show the behavior of the solution quality, which is represented by the inconsistency measure $\mathcal{I}_{2}$, as the iterations of the PSO-based solver increase. (An improvement of the solution quality is graphically represented by a decreasing of $\mathcal{I}_{2}$.) In particular: Fig. 1 plots $\mathcal{I}_{2}$ vs. the PSO iterations in the case where all the indicators of Table 1 are considered and $\mathbf{w}$ are the decision variables; Fig. 2 plots $\mathcal{I}_{2}$ vs. the PSO iterations in the case where only the Altman's variables of Table 3 are considered and both $\mathbf{w}$ and $\mathbf{q}$ are the decision variables.

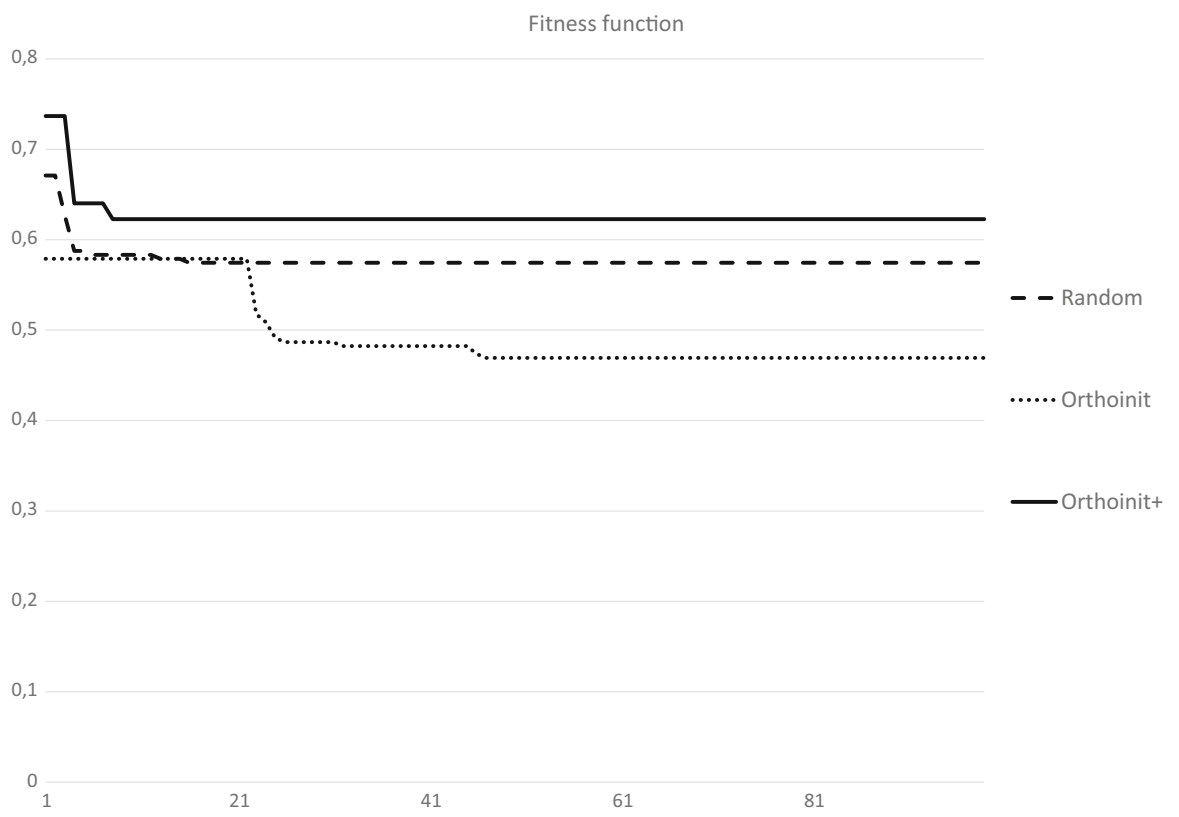

Fig. 1 Values of $\mathcal{I}_{2}(\mathbf{w})$, for the first 100 iterations of the PSO procedures 


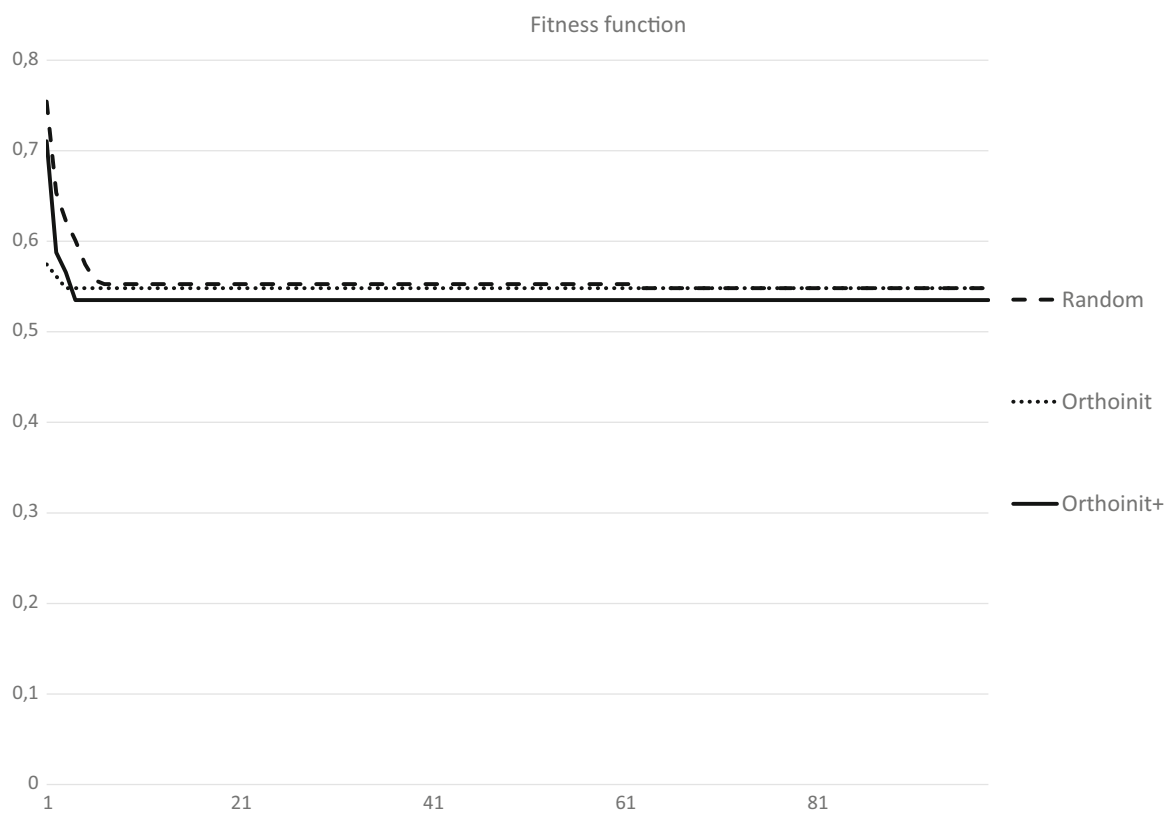

Fig. 2 Values of $\mathcal{I}_{2}(\mathbf{w}, \mathbf{q})$, for the first 100 iterations of the PSO procedures

From these figures, it can be noted that:

- Especially when using the initialization procedure Orthoinit+, a fast decrease of $\mathcal{I}_{2}$ occurs after the very first iterations of PSO, that is when the effects of the uniformly linearly independent choice of the initialization of the PSO particles are still relevant;

- Then, further improvements of the solution quality generally require an additional number of PSO iterations. This could mean that the evolutionary process might be entangled in a plateau of $\mathcal{I}_{2}$. Notice that it happens regardless of the chosen initialization procedure;

- Anyway, the quality of the results achieved within a moderate number of PSO iterations (see the previous tables) may be considered satisfactory for practical applications. This is a positive feature of our MCDA-based method, since the search of a definitely acceptable solution might require a really heavy computational burden.

\section{Comparisons and some proposals}

In this section, we first compare the above results with those coming from the use of a PSO solver based on the penalty function approach, already proposed and adopted in Corazza et al. (2013, 2015b). Then, we propose and apply some new inconsistency measures which are possibly able to jointly use the informative contents from both the bankrupt firms and the nonbankrupt ones. 


\subsection{The comparison with the penalty function approach}

The results presented in Tables 5, 6, 7, 8, 9, 10, 11 and 12 are obtained applying PSO to a (novel) nonlinear reformulation of the mathematical programming problem (8). In this subsection, we compare these results with those got solving the same problem by using again PSO but once (8) has been reformulated on the basis of a penalty function approach (see Subsect. 5.2). Unlike the nonlinear reformulation approach presented above, using a penalty function requires the preliminary setting of an important parameter, the so-called penalty parameter $\epsilon$. In this regard, recalling that the latter approach has been (originally) used for solving a creditworthiness problem in Corazza et al. (2015b) by setting $\epsilon$ to 1 , here we adopt the same value for consistency reasons.

In Tables 13, 14, 15, 16, 17 and 18, we report the results, respectively, corresponding to those shown in Tables 5, 6, 7, 8, 9 and 10. Notice that, with reference to the creditworthiness classification, throughout this paper we considered eighteen different configurations of the type: "Initialization procedure (Random, Orthoinit, Orthoinit+)_Inconsistency measure $\left(\mathcal{I}_{1}, \mathcal{I}_{2}\right)$ - Criteria (all indicators of Table 1, Altman's variables of Table 3 )-Decision variables (w, w and $\mathbf{q})$." The importance for the reader of the last note will become clearer in a while.

Finally, with reference to the creditworthiness classification, from the results in Tables 13, 14, 15, 16, 17 and 18 we can infer that:

- The endogenous determination of the MURAME parameters obtained applying the penalty function approach allows improvements in terms of the percentages of bankrupt firms classified in the best creditworthiness class, with respect to the exogenous specification of the same parameters, only in 9 over 18 of the considered configurations. On the contrary, when using the nonlinear reformulation approach, this percentage reaches its maximum value, i.e. in 18 cases over 18 ;

- The same endogenous determination of the MURAME parameters permits improvements in terms of the percentages of bankrupt firms classified in the worst creditworthiness class, with respect to the exogenous specification of the same parameters, in 10 over 18 configurations. Differently, when using the nonlinear reformulation approach, this performance increases, yielding a satisfactory result in 13 cases over 18 ;

- The obtained creditworthiness classifications fail in satisfying the general principle stated above in 14 over 18 of the considered configurations, even if in some cases the displacement is minimal. Conversely, when using the nonlinear reformulation approach, the performance decreases up to 6 over 18 .

With reference to the endogenously determined parameters, adopting the penalty function approach we can carry out the following conclusions from our experiments:

- Some of the parameters may get negative values (which are not negligibly small), and one weight assumes a value which is even considerably greater than $100 \%$;

- In correspondence with some configurations the summation of the weights may significantly differ from $100 \%$. 


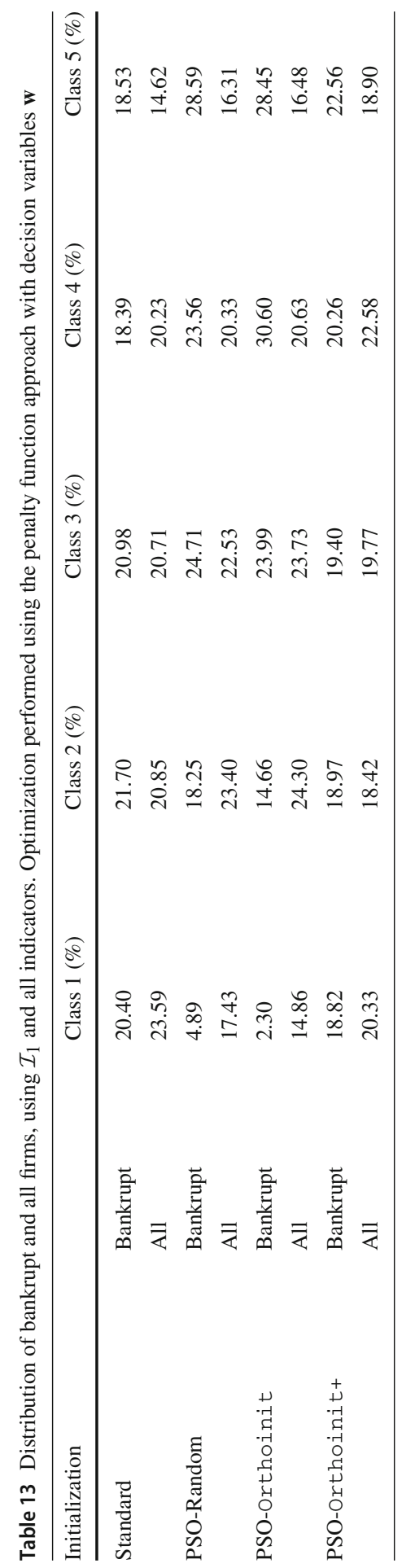




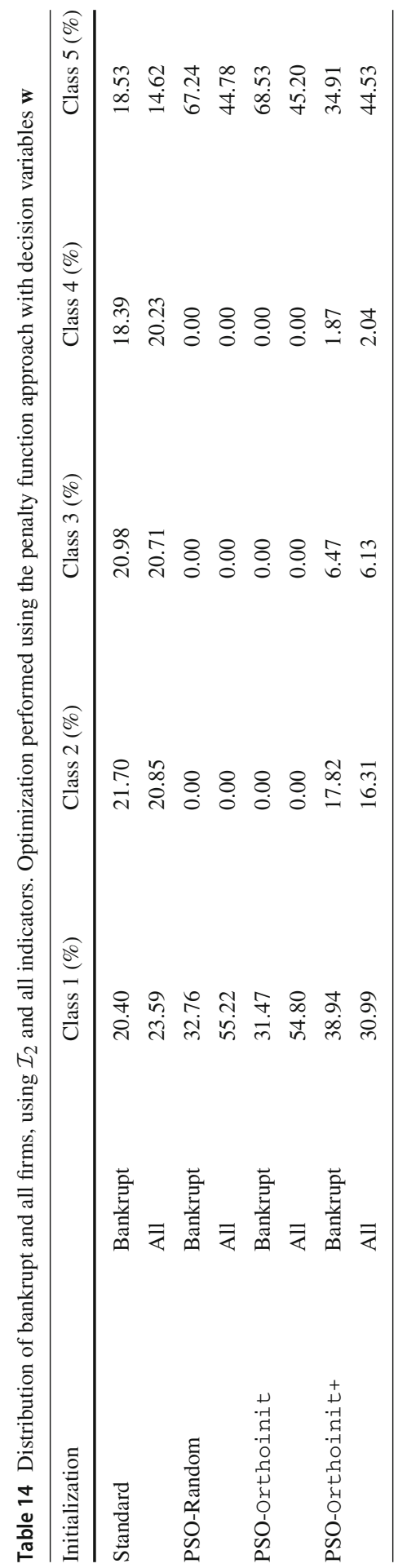




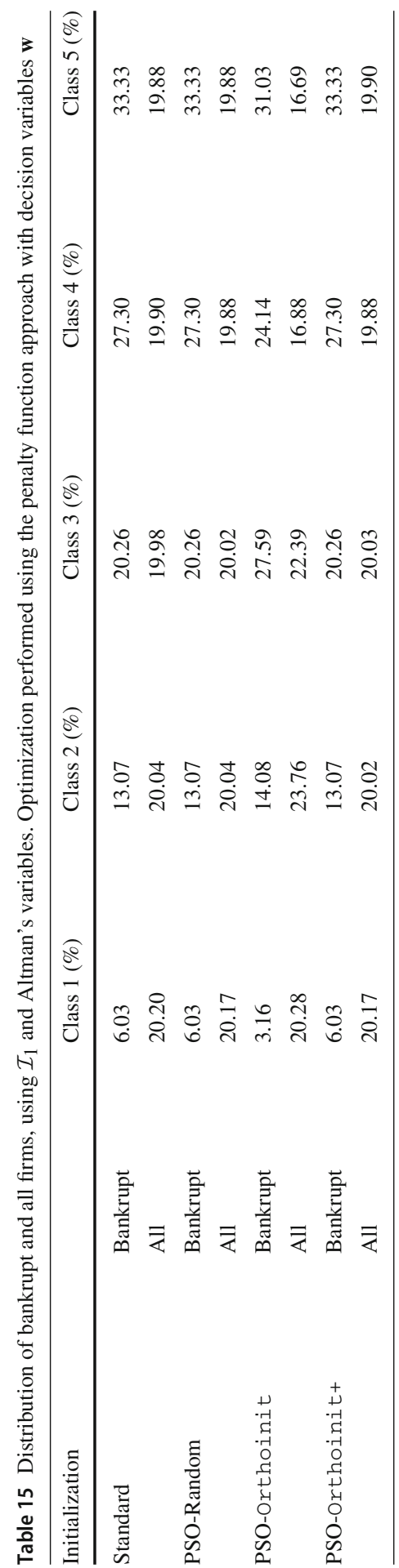




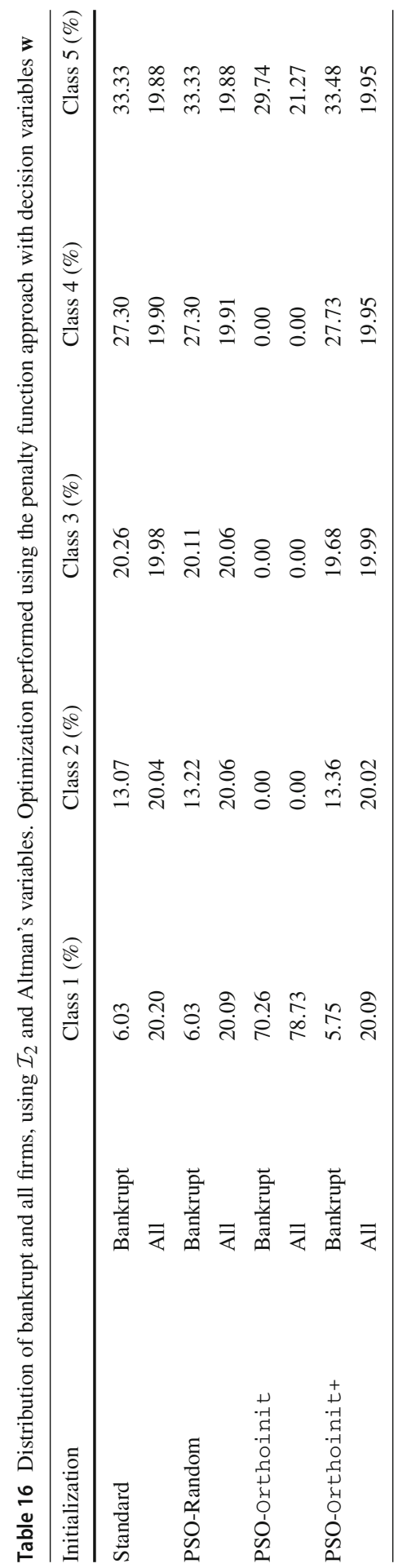




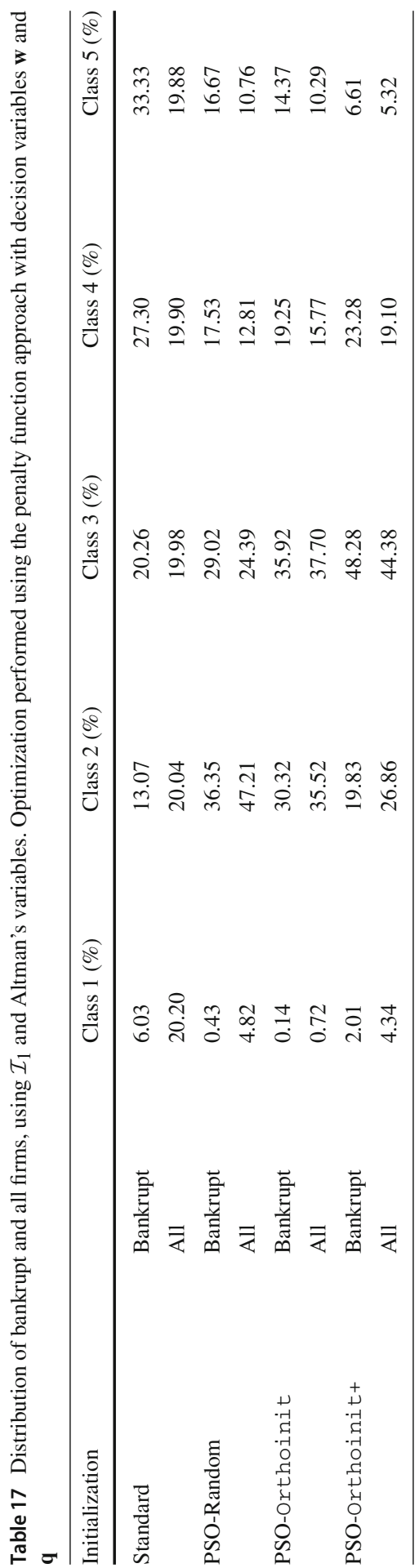




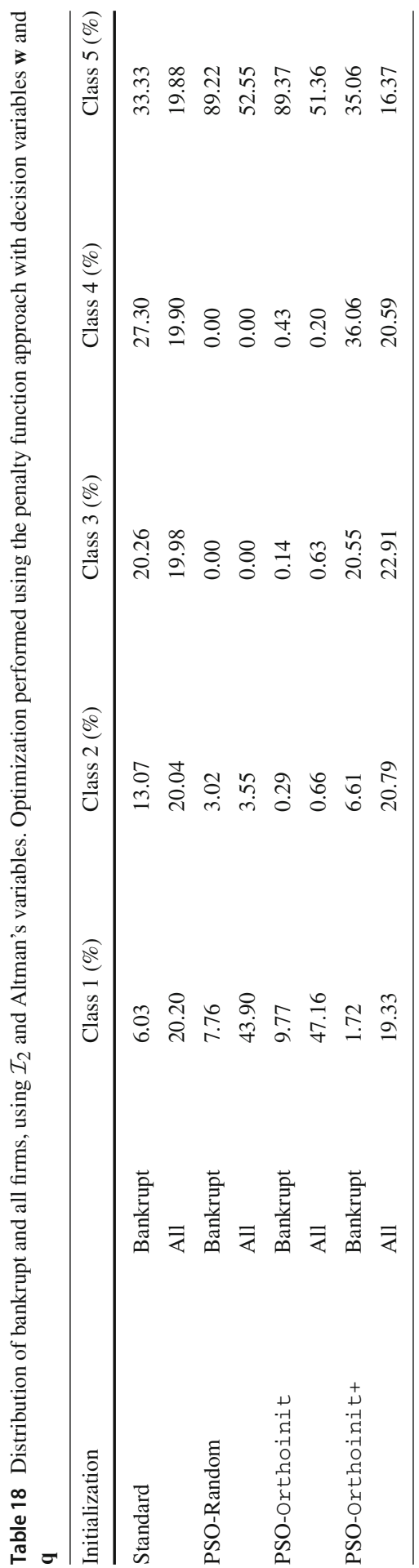


Both the last items reveal a violation of some constraints in (8). Thus, the penalty function approach we adopted seems unable to avoid the generation of infeasible solutions, unlike the nonlinear reformulation.

\subsection{A tentative use of the nonbankrupt firms' informative content}

As discussed in Sect. 4, the use of the nonbankrupt firms' informative content for the endogenous determination of the weights $\left\{w_{j}\right\}$ and possibly of the indifference thresholds $\left\{q_{j}\right\}$ yet represents a challenging task.

Although this issue is not central in the current study, we nevertheless advance some very preliminary ideas that could properly be investigated in future researches. In particular, in this subsection: first we propose some simple extensions of the inconsistency measures (9), which are possibly able to manage the information contained even in the nonbankrupt firms; then we apply these new measures to the classification of the bankrupt firms (we recall that the current work is focused on the correct classification of the latter kind of firms); lastly we compare the obtained results with those coming from our MURAME-based creditworthiness evaluation model.

As far as the specification of the new (very preliminary) inconsistency measures regards, we still consider the general principle set out in Subs. 4.1 related to bankrupt firms, here accompanied by the following similar general principle concerning the nonbankrupt firms: a good creditworthiness classification model should place as many nonbankrupt firms as possible in the best rating class, and the number of nonbankrupt firms in each class should decrease as the class creditworthiness quality decreases. So, denoting by $n_{j}^{N B}$ the number of nonbankrupt firms classified in the $j$-th class and by $N^{N B}$ the total number of nonbankrupt firms, according to both the aforementioned principles, we suggest the following two new inconsistency measures:

$$
\begin{aligned}
\widetilde{\mathcal{I}}_{1}\left(w_{1}, \ldots, w_{n}\right) & \doteq \widetilde{\mathcal{I}}_{1}=\lambda \mathcal{I}_{1}+(1-\lambda) \frac{n_{5}^{N B}}{N^{N B}}=\lambda \frac{n_{1}^{B}}{N^{B}}+(1-\lambda) \frac{n_{5}^{N B}}{N^{N B}} \\
\tilde{\mathcal{I}}_{2}\left(w_{1}, \ldots, w_{n}\right) & \doteq \widetilde{\mathcal{I}}_{2}=\lambda \mathcal{I}_{2}+(1-\lambda)\left(1-\frac{n_{1}^{N B}}{N^{N B}}\right) \\
& =1-\left[\lambda \frac{n_{5}^{B}}{N^{B}}+(1-\lambda) \frac{n_{1}^{N B}}{N^{N B}}\right],
\end{aligned}
$$

where $\lambda \in[0,1]$ is a parameter indicating the preference of the DM toward the informative content coming from the bankrupt firms.

As for the applications of these new measures to the classification of bankrupt firms, we turn our attention only to those configurations that our numerical experience generally led to the best classification results, that is: Random and Orthoinit+ as initialization procedures; Altman's variables as criteria; $\mathbf{w}$ and $\mathbf{q}$ as decision variables. Lastly, having no argumentations to prefer bankrupt firms' informative content to the nonbankrupt firms' one and vice versa, we set $\lambda=0.5$.

Tables 19 and 20 report the results using $\widetilde{\mathcal{I}}_{1}$ and $\widetilde{\mathcal{I}}_{2}$, and represent the counterpart of Tables 9 and 10, respectively. 


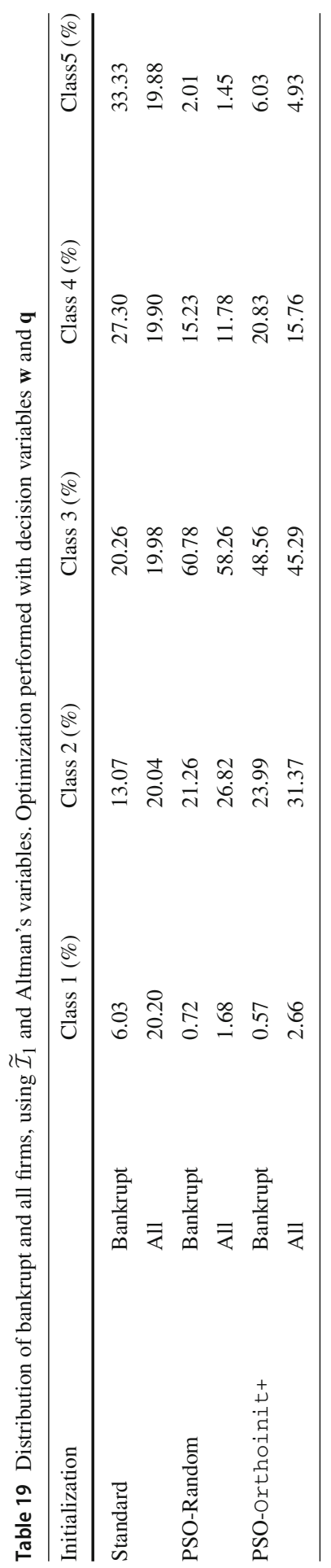




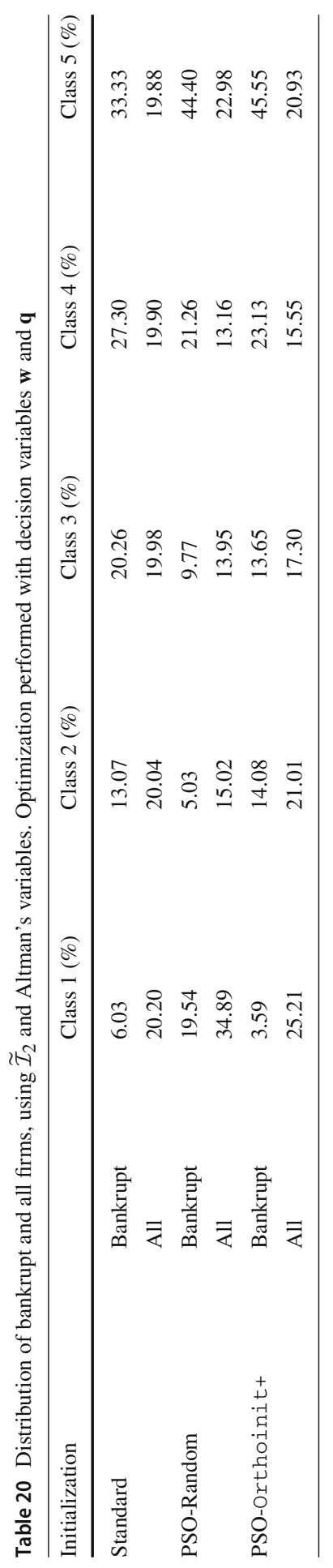


From the results reported in these tables, it can be noted that:

- The overall quality of the creditworthiness distribution of bankrupt firms depends on the inconsistency measure applied. Indeed, in the case of $\widetilde{\mathcal{I}}_{1}$, the obtained classifications heavily dissatisfy the general principle concerning the bankrupt firms themselves, regardless of the initialization procedures (see Table 19). Conversely, this principle dissatisfaction is less evident when using $\widetilde{\mathcal{I}}_{2}$ and almost disappears when applying the initialization procedure Orthoinit+ (see Table 19). These findings confirm some of the general conclusions already presented. Indeed, in terms of creditworthiness classification: $\widetilde{\mathcal{I}}_{2}$, which derives from $\mathcal{I}_{2}$, seems preferable to $\widetilde{\mathcal{I}}_{1}$, which derives from $\mathcal{I}_{1}$, as $\mathcal{I}_{2}$ resulted preferable to $\mathcal{I}_{1}$; Orthoinit+ generally performs better than Random;

- The endogenous determination of the MURAME parameters based on $\tilde{\mathcal{I}}_{1}$ and on $\widetilde{\mathcal{I}}_{2}$, respectively, leads to a quality of the bankrupt firms' creditworthiness distributions which is not much worse than the one of the corresponding distributions achieved through the application of $\mathcal{I}_{1}$ and of $\mathcal{I}_{2}$, respectively;

- The (sub-)optimal values of the weights and of the indifference thresholds (not present here) do not show noteworthy features and, in any case, not particularly different from those detectable by the corresponding results achieved when using $\mathcal{I}_{1}$ and $\mathcal{I}_{2}$, respectively.

The second item is notably interesting. It highlights that the new inconsistency measures appear to perform in a way almost comparable with the one of their ancestors (9). In its turn, this implicitly underlines the role played by the trade-off between the quality of the classification results and the amount of computational time. (Notice that this trade-off characterizes our evolutionary approach for the creditworthiness evaluation.) Indeed, on one hand, the above finding promotes to deepen the research on $\widetilde{\mathcal{I}}_{1}$ - and $\widetilde{\mathcal{I}}_{2}$-like inconsistency measures in order to improve the quality of the obtained results. On the other hand, such a further research would require greater computational efforts than those related to the measures of inconsistency (9).

\section{Conclusions and future work}

In this paper, we have proposed a MCDA-based approach for the creditworthiness evaluation of Italian SMEs. It allows to simultaneously consider as criteria several different indicators, derived from balance sheet data of the firms, and provides results in terms of classification of the firms in homogeneous rating classes. Our proposal is characterized by the use of three distinctive elements, being all them relevant to our purposes. First, we definitely privileged an endogenous computation of some MURAME parameters, with a specific emphasis on the application in hand. Second, similarly to what done in Corazza et al. (2015b), we adopted a PSO-based scheme to solve an appropriate constrained optimization problem, which is related to the endogenous determination of MURAME parameters. However, we gained effectiveness and efficiency ignoring the penalty approach in Corazza et al. (2015b), which might cause possible instabilities, and introducing a nonlinear change of the involved unknowns which could better exploit the simplex-like structure of the feasible set of the afore- 
mentioned problem. Third, unlike (Corazza et al. 2015b), we endowed PSO with a couple of specific deterministic initializations, which are tailored on our problem of classifying performance for bankrupt firms, as numerical results confirmed. Moreover, the overall PSO scheme we adopted was able to early generate reasonably good (sub-)optimal solutions.

In the future, we plan to possibly extend the study of the efficient determination of MURAME parameters, by endogenously assessing not only the weights and the indifference thresholds associated with the criteria, but also all MURAME parameters.

Acknowledgements G. Fasano thanks the National Research Council-Marine Technology Research Institute (CNR-INSEAN), Italy, for the support received. G. Fasano also wishes to thank the working group GNCS of IN $\delta$ AM (Istituto Nazionale di Alta Matematica).

Funding Open access funding provided by Università Ca' Foscari Venezia within the CRUI-CARE Agreement.

Open Access This article is licensed under a Creative Commons Attribution 4.0 International License, which permits use, sharing, adaptation, distribution and reproduction in any medium or format, as long as you give appropriate credit to the original author(s) and the source, provide a link to the Creative Commons licence, and indicate if changes were made. The images or other third party material in this article are included in the article's Creative Commons licence, unless indicated otherwise in a credit line to the material. If material is not included in the article's Creative Commons licence and your intended use is not permitted by statutory regulation or exceeds the permitted use, you will need to obtain permission directly from the copyright holder. To view a copy of this licence, visit http://creativecommons.org/licenses/by/4.0/.

\section{Appendix}

In this appendix, we provide a brief summary of the theoretical results supporting the deterministic PSO initialization procedures in Sect. 5.

Let us assume that $\mathbf{r}_{l, 1}^{k}=r \mathbf{1}, \mathbf{r}_{l, 2}^{k}=r_{g} \mathbf{1}$, with given $r, r_{g}>0$ and $\mathbf{1}=(1, \ldots, 1)^{T} \in$ $\mathbb{R}^{n}$, that is PSO iteration (10)-(11) becomes deterministic. Then, assume that for any $k>0$ we set $w^{k}=w>0, \chi^{k}=\chi>0$, and that the following relations hold

$$
\left\{\begin{array}{l}
a=\chi w<1 \\
\omega=\chi\left(c_{1} r+c_{2} r_{g}\right)<2(\chi w+1) \\
\omega \neq(1 \pm \sqrt{\chi w})^{2}
\end{array}\right.
$$

which ensure necessary conditions to avoid diverging particles trajectories (see Campana et al. 2010). Under the latter conditions, denoting with

$$
X_{l}(k)=\left(\begin{array}{c}
\mathbf{v}_{l}^{k} \\
\mathbf{x}_{l}^{k}
\end{array}\right) \in \mathbb{R}^{2 n}
$$

the state of particle $l$ at iteration $k$, it is easy to prove that the update (10)-(15) can be rewritten as the dynamic linear system

$$
X_{l}(k+1)=\mathcal{A} X_{l}(k)+\mathcal{B}(k),
$$


with

$$
\mathcal{A}=\left(\begin{array}{cc}
a I & -\omega I \\
a I & (1-\omega) I
\end{array}\right) \in \mathbb{R}^{2 n \times 2 n} .
$$

In particular, we can write

$$
X_{l}(k)=X_{l}^{L}(k)+X_{l}^{F}(k)
$$

where $X_{l}^{L}(k)$ is the so-called free response and has the representation

$$
X_{l}^{L}(k)=\mathcal{A}^{k} X_{l}(0),
$$

so that $X_{l}^{L}(k)$ is independent of $\mathbf{p}_{l}^{k}$ and $\mathbf{p}_{g}^{k}$ (see again Campana et al. 2010).

Since the free response depends only on the initial state of the particle, an appropriate choice of the initial state can force $X_{l}^{L}(k)$ to retain specific properties. In particular, when (24) holds it is possible to prove that $\mathcal{A}$ has only two distinct eigenvalues $\lambda_{1}$ and $\lambda_{2}$. Now, let us consider, for a given $k \geq 0$, the quantities

$$
\gamma_{1}(k)=\frac{\lambda_{1}^{k}\left(a-\lambda_{2}\right)-\lambda_{2}^{k}\left(a-\lambda_{1}\right)}{\lambda_{1}-\lambda_{2}}, \quad \gamma_{2}(k)=\frac{\omega\left(\lambda_{1}^{k}-\lambda_{2}^{k}\right)}{\lambda_{1}-\lambda_{2}}
$$

and the $2 n$ vectors in $\mathbb{R}^{2 n}$

$$
\begin{gathered}
z_{i}(k)=\left(\begin{array}{c}
\frac{\gamma_{2}(k)}{\gamma_{1}(k)} e_{i} \\
e_{i}
\end{array}\right), \quad i=1, \ldots, n \\
z_{n+i}(k)=\left(\begin{array}{c}
-\frac{\gamma_{1}(k)}{\gamma_{2}(k)} e_{i} \\
e_{i}
\end{array}\right), \quad i=1, \ldots, n,
\end{gathered}
$$

where $e_{i} \in \mathbb{R}^{n}$ represents the $i$-th unit vector. Finally, the vectors in (29) can be used to set PSO initializations, as detailed in Sect. 5.

\section{References}

Akkoç, S.: An empirical comparison of conventional techniques, neural networks and the three stage hybrid Adaptive Neuro Fuzzy Inference System (ANFIS) model for credit scoring analysis: the case of Turkish credit card data. Eur. J. Oper. Res. 222, 168-178 (2012)

Altman, E.I., Sabato, G.: Effects of the new Basel capital accord on bank capital requirements for SMEs. J. Financ. Serv. Res. 28, 15-42 (2005)

Altman, E.I., Sabato, G.: Modelling credit risk for SMEs: evidence from the US market. ABACUS 43, 332-357 (2007)

Altman, E.I., Marco, G., Varetto, F.: Corporate distress diagnosis: Comparisons using linear discriminant analysis and neural networks (the Italian experience). J. Bank. Finance 18, 505-529 (1994) 
Altman, E.I., Sabato, G., Wilson, N.: The value of non-financial information in small and medium-sized enterprise risk management. J. Credit Risk 6, 1-33 (2010)

Altman, E.I., Esentato, M., Sabato, G.: Assessing the credit worthiness of Italian SMEs and mini-bond issuers. Global Finance J. 43, 100450 (2020)

Angilella, S., Mazzú, S.: The financing of innovative SMEs: a multicriteria credit rating model. Eur. J. Oper. Res. 244, 540-554 (2015)

Angilella, S., Mazzú, S.: A credit risk model with an automatic override for innovative small and mediumsized enterprises. J. Oper. Res. Soc. 70, 1784-1800 (2019)

Baourakis, G., Coniescu, M., Van Dijk, G., Pardalos, P.M., Zopounidis, C.: A multicriteria approach for rating the credit risk of financial institutions. CMS 6, 347-356 (2009)

Basel Committee on Banking Supervision: International convergence of capital measurement and capital standards-a revised framework. Basel Committee on Banking Supervision, Basel (2004)

Belton, V., Stewart, T.J.: Multiple criteria decision analysis: an integrated approach. Springer, Berlin (2002)

Blackwell, T., Kennedy, J., Poli, R.: Particle swarm optimization: an overview. Swarm Intell. 1, 33-57 (2007)

Bonabeau, E., Dorigo, M., Theraulaz, G.: From Natural to Artificial Swarm Intelligence. Oxford University Press, Oxford (1999)

Brans, J.P., Vincke, P.A.: A preference ranking organization method: the Promethee method for multiple criteria decision making. Manag. Sci. 31, 647-656 (1985)

Campana, E.F., Fasano, G., Pinto, A.: Dynamic analysis for the selection of parameters and initial population, in particle swarm optimization. J. Global Optim. 48, 347-397 (2010)

Corazza, M., Di Tollo, G., Fasano, G., Pesenti, R.: A novel initialization of PSO for costly portfolio selection problems. Department of Management, Ca' Foscari University of Venice-Working Papers, 2015/04, pp. 1-27 (2015a)

Corazza, M., Fasano, G., Gusso, R.: Particle Swarm Optimization with non-smooth penalty reformulation, for a complex portfolio selection problem. Appl. Math. Comput. 224, 611-624 (2013)

Corazza, M., Funari, S., Gusso, R.: An evolutionary approach to preference disaggregation in a MURAMEbased creditworthiness problem. Appl. Soft Comput. 29, 110-121 (2015b)

Corazza, M., Funari, S., Gusso, R.: Creditworthiness evaluation of Italian SMEs at the beginning of the 2007-2008 crisis: an MCDA approach. The North Am. J. Econ. Finance 38, 1-26 (2016)

Corazza, M., Funari, S., Siviero, F.: A MURAME-based technology for bank decision support in creditworthiness assessment. Banks Bank Syst. 9, 7-17 (2014)

Cura, T.: Particle swarm optimization approach to portfolio optimization. Nonlinear Anal. Real World Appl. 10, 2396-2406 (2009)

Diez, M., Serani, A., Leotardi, C., Campana, E.F., Fasano, G., Gusso, R.: Dense orthogonal initialization for deterministic PSO: ORTHOinit+. The seventh International Conference on Swarm Intelligence (IC-SI 2016) [Lecture Notes in Computer Science 9713, Part I], Springer, pp. 322-330 (2016)

Doumpos, M., Figueira, J.R.: A multicriteria outranking approach for modeling corporate credit ratings: an application of the Electre Tri-nC method. Omega 82, 166-180 (2019)

Doumpos, M., Pasiouras, F.: Developing and testing models for replicating credit ratings: a multicriteria approach. Comput. Econ. 25, 327-341 (2005)

Doumpos, M., Zopounidis, C.: A multicriteria outranking modeling approach for credit rating. Decis. Sci. 42, 721-742 (2011)

Doumpos, M., Kosmidou, K., Baourakis, G., Zopounidis, C.: Credit risk assessment using a multicriteria hierarchical discrimination approach: a comparative analysis. Eur. J. Oper. Res. 138, 392-412 (2002)

Fawcett, T.: An introduction to ROC analysis. Pattern Recogn. Lett. 27, 861-874 (2006)

García, F., Giménez, V., Guijarro, F.: Credit risk management: a multicriteria approach to assess creditworthiness. Math. Comput. Modell. 57, 2009-2015 (2013)

Goletsis, Y., Askounis, D.T., Psarras, J.: Multicriteria judgements for project ranking: an integrated methodology. Econ. Financ. Model. 8, 127-148 (2001)

Goletsis, Y., Psarras, J., Samoulidis, J.E.: Project ranking in the Armenian energy sector using a multicriteria method for groups. Ann. Oper. Res. 120, 135-157 (2003)

Hand, D.J., Till, R.J.: A simple generalisation of the area under the ROC curve for multiple class classification problems. Mach. Learn. 45, 171-186 (2001)

Jacquet-Lagrèze, E., Siskos, Y.: Preference disaggregation: 20 years of MCDA experience. Eur. J. Oper. Res. 130, 233-245 (2001) 
Kadzinski, M., Martyn, K., Cinelli, M., Slowinski, R., Corrente, S., Greco, S.: Preference disaggregation for multiple criteria sorting with partial monotonicity constraints: application to exposure management of nanomaterials. Int. J. Approx. Reason. 117, 60-80 (2020)

Kadzinski, M., Ghaderi, M., Dabrowski, M.: Contingent preference disaggregation model for multiple criteria sorting problem. Eur. J. Oper. Res. 281(2), 369-387 (2020)

Kennedy, J., Eberhart, R.C.: Particle swarm optimization. In: Proceedings of the IEEE International Conference on Neural Networks IV pp. 1942-1948 (1995)

Khalil, J., Martel, J.M., Jutras, P.: A multicriterion system for credit risk rating. Gestion 2000: Belgian Manag. Mag. 15, 125-146 (2000)

Marqués, A.I., García, V., Sánchez, J.S.: A literature review on the application of evolutionary computing to credit scoring. J. Oper. Res. Soc. 64, 1384-1399 (2013)

Mousavi, M.M., Ouenniche, J.: Multi-criteria ranking of corporate distress prediction models: empirical evaluation and methodological contributions. Ann. Oper. Res. 271, 853-886 (2018)

Roy, B.: Classement et choix en presence de criteres multiples (la méthode ELECTRE). RIRO 8, 57-75 (1968)

Serani, A., Leotardi, C., Iemma, U., Campana, E.F., Fasano, G., Diez, M.: Parameter selection in synchronous and asynchronous deterministic particle swarm optimization for ship hydrodynamics problems. Appl. Soft Comput. 49, 313-334 (2016)

Waegeman, W., De Baets, B., Boullart, L.: ROC analysis in ordinal regression learning. Pattern Recognit. Lett. 29, 1-9 (2008)

Zhang, W.J., Xie, X.F., Bi, D.C.: Handling boundary constraints for numerical optimization by particle swarm flying in periodic search space. Proceedings of the 2004 IEEE Congress on Evolutionary Computation, IEEE, pp. 2307-2311 (2005)

Publisher's Note Springer Nature remains neutral with regard to jurisdictional claims in published maps and institutional affiliations. 